\title{
A Three-Dimensional Fractal Contact Model for a Rough Friction Surface With Multiple-Scale Asperities
}

\author{
Ao Liang \\ Tongji University \\ Yongming Bian ( $\square$ li-ang86@163.com) \\ Tongji University \\ Guangjun Liu \\ Tongji University
}

\section{Research Article}

Keywords: Three-dimensional friction surface, multiple-scale level, asperity, fractal theory

Posted Date: May 28th, 2021

DOI: https://doi.org/10.21203/rs.3.rs-527873/v1

License: (c) (i) This work is licensed under a Creative Commons Attribution 4.0 International License.

Read Full License 


\title{
A Three-dimensional Fractal Contact Model for a Rough Friction Surface with Multiple-scale Asperities
}

\author{
Ao Liang ${ }^{1}$, Yongming Bian ${ }^{1 *}$, Guangjun Liu ${ }^{1}$ \\ (1 School of Mechanical Engineering, Tongji University, Shanghai 201804, China) \\ *Corresponding author: ymbianmail@163.com
}

\begin{abstract}
:
This paper describes a new fractal contact model for a rough three-dimensional friction surface considering scale dependence. The model predicts the total contact area as a function of contact load. The microcontact fractal model for asperities at multiple-scale levels is derived. The truncated area distribution function of asperities at multiple-scale levels is revised. The effects of each scale level and fractal parameters on the proposed model are evaluated by numerical simulations. Results obtained from the complex fractal model with several scales are accurate as demonstrated by comparing them to experimental results and models taken from literature. Friction and wear of rough surfaces can be further studied using the proposed model.
\end{abstract}

Key words: Three-dimensional friction surface; multiple-scale level; asperity; fractal theory

\section{Introduction}

Establishing and analyzing a contact model for a rough three-dimensional friction surface is helpful for researching the friction, wear and contact fatigue of mechanical components [1-3]. From a microscopic perspective, rough surfaces of mechanical parts have a large number of asperities of different sizes. When two rough friction surfaces are loaded, it is primarily their asperity summits that come in contact, i.e., the real contact area is smaller than the nominal contact area. The contacting asperities on the rough surfaces support high compressive stresses, which can cause material yielding. Therefore, establishing an effective contact model is essential for evaluating the capacity of friction surfaces to carry loads, improving surface abrasion resistance, and extending the service life of friction components.

From a microscopic point of view, the main models used to research rough surface contact problems are finite element models, statistical models, and fractal theory models. The finite element models use numerical algorithms to obtain directly the contact stress field on the rough surface and the relationship between the actual contact area and the normal contact load [4-7]. However, the elastoplastic character of contact between different materials or rough surfaces with larger sample length makes modeling 
challenging and increases computational cost. Sometimes, it may even be necessary to write additional complex programming scripts for further analysis. Statistical models and fractal theory models for rough surfaces [8] use theoretical derivations to obtain analytical formulas for the contact area and contact load, which makes modelling easier. The earliest models adopted to research the contact characteristics of rough surfaces are statistical models. Greenwood and Williamson [9] developed a classical contact mechanics model (the GW model) using statistical theory. This model assumes that the radius of curvature of all asperities on the rough surface is the same and the height distribution of asperities is represented by a statistical probability density function. Therefore, an analytical formula for the contact load and actual contact area can be obtained. Various extensions of the GW contact model, such as the Chang-Etsion-Bogy (CEB) model [10], Zhao-Maietta-Chang (ZMC) model [11], Kogut-Etsion (KE) model [12], Cohen-Kligerman-Etsion (CKE) model [13], complete elastic model [14], and sizedependent plasticity model [15], have been proposed to incorporate the influences of elastic and plastic deformations. Although these models can approximately solve the problems encountered in practical engineering, their statistical parameters depend on the sampling length and the resolution of the measuring instruments, resulting in nonunique results of the characterization and analysis of rough surfaces.

While trying to overcome the shortcomings of statistical models, many researchers found by studying microscopic characteristics of contact surfaces that self-affinity and self-similarity are present on the rough surfaces at various measurement scales [16]. Therefore, the fractal geometry theory was widely adopted to describe surface topographies. Majumdar and Bhushan [17] first proposed a twodimensional surface fractal contact model (the MB model) to analyze the actual contact area as a function of load using the Weierstrass-Mandelbrot function (the WM function). Considering the influence of friction on the asperities of the friction surface, Wei et al. [18] used a cubic polynomial to represent the mechanical contact properties of an asperity subjected to elastoplastic deformations and developed a contact model using a two-dimensional fractal theory to investigate the influence of friction coefficient and the fractal dimension of an actual contact area. Yan and Komvopoulos [19] proposed a modified two-variable WM function, and created for the first time a three-dimensional surface contact model (YK model), by obtaining the relationships between surface topography parameters, material properties and total contact load. Using the YK model, Liu et al. [20] established a modified fractal contact model considering friction. However, the results from the model revealed that small contact spots of a rough surface exhibited plastic deformation, while the large contact spots were in elastic the deformation range. This unusual behavior was contrary to the predictions of the classical contact mechanics and may affect many tribological properties, such as wear, adhesion, and friction. Thus, Morag and Etsion [21] established a contact model for a single asperity which transitioned from elastic to plastic contact (the ME model) by modifying the MB model. The ME model demonstrated that the critical microcontact 
area for a single asperity was scale-dependent. Liou and Lin [22] revised the YK model by redefining the elastoplastic inception and the fully plastic regime inception. Virtually all engineering surfaces exhibit some multiple-scale fractal characteristics [23]. Miao [24] proposed a complete fractal contact model by extending the ME model. However, the scale dependence is not mentioned in the fractal contact model considering friction. Based on the three-dimensional fractal theory, Pan [25] proposed a fractal load model for a rough friction surface considering elastoplastic contact between asperities and compared it to experimental data and other models to verify its validity. Wang [26] established a loadingunloading three-dimensional rough fractal spherical contact model for friction and obtained the actual contact area as a function of load during the loading and unloading process at different deformation stages. In the above two models, the critical microcontact area of asperities is scale-independent and does not change with the geometric dimensions of the asperities.

new three-dimensional elastoplastic contact model for rough friction surfaces considering scale dependence is presented herein based on the fractal theory. The formulas for critical contact area and critical deformation conditions for asperities at different scales are derived. The area distribution functions of asperities of various scale levels are derived and the analytical expressions for the total actual contact area and the normal contact load are proposed. Finally, the applicability and rationality of the proposed model are verified by comparing it to other models and experimental data, which reveals that the present model is more consistent with the actual physical behavior.

\section{Characterization of three-dimensional rough surface}

The three-dimensional morphology of rough surface can be expressed using the WM function [27], which adopts a superposition of cosine functions of different wavelengths. WM function is commonly used to generate random fractal surfaces [28-30]. The two-variable WM function proposed by Yan and Komvopoulos for a three-dimensional surface is as follows [19]:

$$
\begin{aligned}
Z(x, y)= & L\left(\frac{G}{L}\right)^{D-2}\left(\frac{\ln \gamma}{M}\right)^{0.5} \times \\
& \sum_{m=1}^{M} \sum_{n=0}^{n_{m a x}} \gamma^{(D-3) n}\left\{\cos \phi_{m, n}-\cos \left[\frac{2 \pi \gamma^{n}\left(x^{2}+y^{2}\right)^{0.5}}{L} \times \cos \left(\tan ^{-1}\left(\frac{y}{x}\right)-\frac{\pi m}{M}\right)+\phi_{m, n}\right]\right\}
\end{aligned}
$$

where $L$ denotes the sample length; $G$ denotes the fractal roughness of the three-dimensional surface; $D \quad(2<D<3)$ represents the fractal dimension; $M$ determines the number of superposed ridges on the surface; $\gamma(\gamma>1)$ denotes a scaling parameter, which is generally chosen as 1.5 ; and $\phi_{m, n}$ represents the random phase $\left(0 \leq \phi_{m, n} \leq 2 \pi\right) ; n$ denotes the frequency level for asperities, where $n_{\max }=\operatorname{int}\left[\log \left(L / L_{h}\right) / \log \gamma\right]$ is the highest level of $n, \operatorname{int}[]$ denotes the integer part of the value in the 
brackets, and $L_{h}$ denotes the shortest length scale determined by the resolution of the measuring instrument used.

To illustrate the physical significance of the parameters used in Eq. (1), selected fractal surface topographies were simulated using various values of $n_{\max }$ and $M$. The highest level of asperities, $n_{\max }$, on rough surfaces is related to the shortest cut-off length, $L_{h}$. Four simulated isotropic rough surfaces with $n_{\max }=2,6,8$, and 20, corresponding to the cut-off length values $L_{h}=0.5 \mathrm{~mm}, 0.1 \mathrm{~mm}, 0.04 \mathrm{~mm}$, and 0.003 $\mathrm{mm}$, for the fixed values of $D=2.3, G=1.5 \times 10^{-10} \mathrm{~mm}, L=1 \mathrm{~mm}$, and $M=6$, are plotted in Fig. 1 . Comparison of these rough surface topographies demonstrates at a given fractal dimension, $D$, fractal roughness, $G$, sample length, $L$, and ridge number, $M$, with the increase in the value of $n_{\max }$, the cut-off length, $L_{h}$, decreases, the scale level of the wave applied to simulate the fractal rough surface steadily increases, and the surface topography becomes more complex. Figure 2 shows the influence of different ridge number values, $M=1,2,4$, and 6 , on the rough surface morphology with fixed parameters $D=2.3$, $G=1.5 \times 10^{-10} \mathrm{~mm}, L=1 \mathrm{~mm}$, and $n_{\max }=34$. It can be seen that with the increase in ridge number, $M$, the number of cosine functions used to describe rough surface increases. When $M=1$, the rough surface is generated by only one cosine wave along the horizontal x-axis, as illustrated in Figure 2(a). Thus, the bivariate WM function can be transformed into the univariate WM function, which simplifies the further development of the contact model. When $M=2$, surface topography is composed of two waves along the horizontal transverse $\mathrm{x}$-axis and the horizontal longitudinal $\mathrm{y}$-axis respectively. The difference among the surfaces illustrated in Figure 2 is elucidated by observing the contour plot of the corresponding power spectra (Figure 3) calculated by fast Fourier transform. 


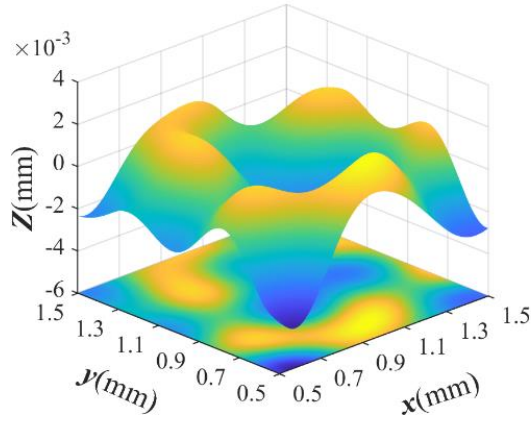

(a) $\mathrm{n}_{\max }=2$

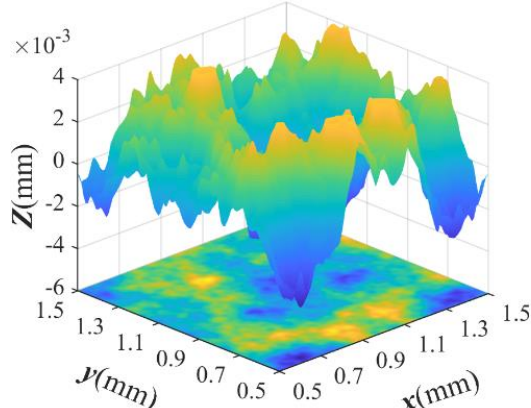

(c) $n_{\max }=8$

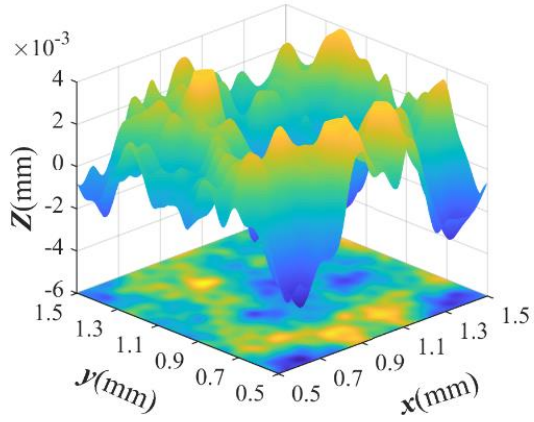

(b) $\mathrm{n}_{\max }=6$

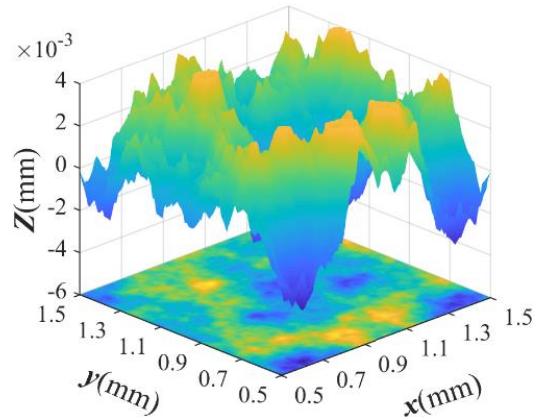

(d) $n_{\text {max }}=20$

Fig. 1 Surface topographies for $D=2.3, G=1.5 \times 10^{-10} \mathrm{~mm}, L=1 \mathrm{~mm}$, and $M=6$

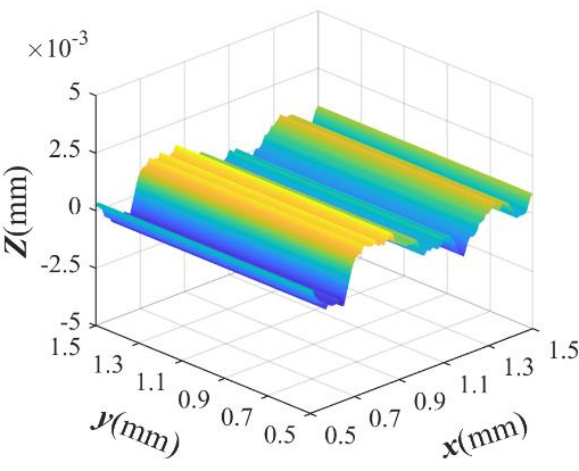

(a) $\mathrm{M}=1$

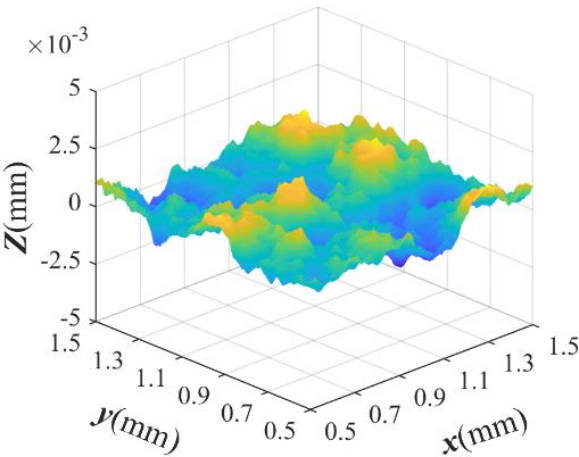

(c) $\mathrm{M}=4$

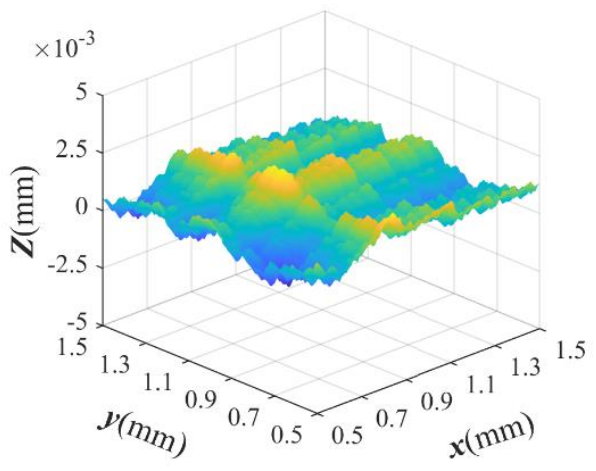

(b) $\mathrm{M}=2$

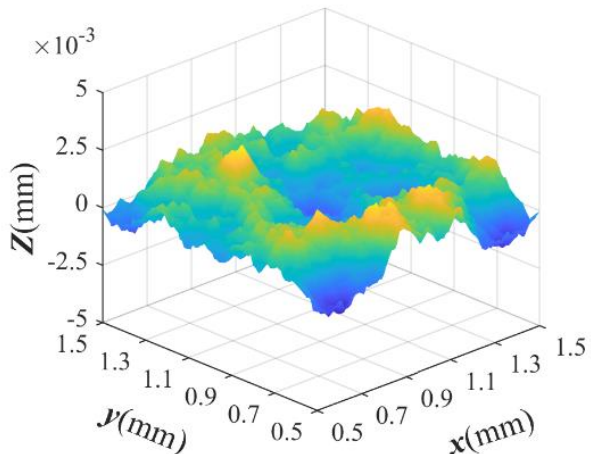

(d) $\mathrm{M}=6$

Fig. 2 Surface topographies for $D=2.3, G=1.5 \times 10^{-10} \mathrm{~mm}, L=1 \mathrm{~mm}$, and $n_{\max }=34$ 


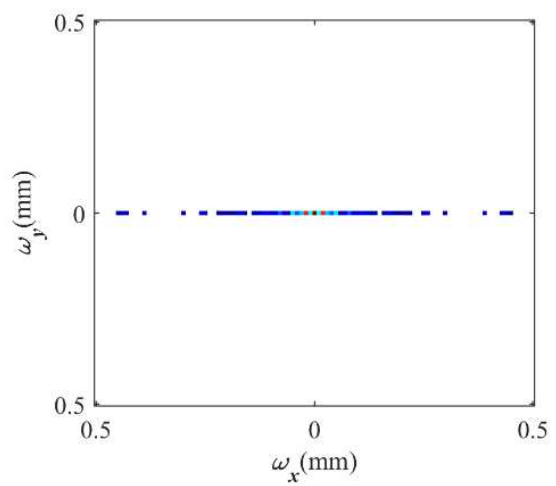

(a) $\mathrm{M}=1$

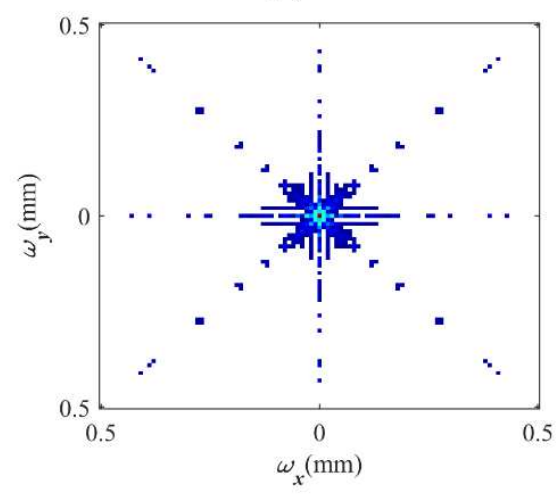

(c) $M=4$

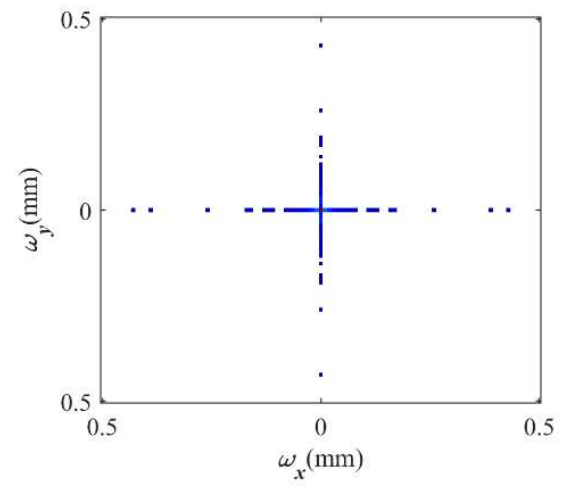

(b) $\mathrm{M}=2$

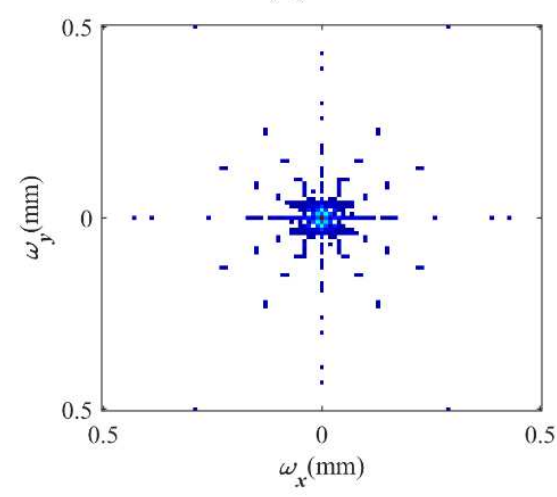

(d) $M=6$

Fig. 3 Contour graphs of fractal surface topography power spectra

\section{Contact model for a single asperity}

In order to study the contact processes between the rough friction surfaces, one of them is modelled as a rigid flat surface in contact with an equivalent rough surface. This section analyses the contact of a single asperity on a rough surface.

Based on the rough surface simulations shown in Figure 2(a), assuming that the number of assumed ridges is equal to $1(M=1)$, the two-variable WM function (Eq. (1)) can be simplified as follows:

$$
Z(x)=L\left(\frac{G}{L}\right)^{(D-2)}(\ln \gamma)^{0.5} \sum_{n=0}^{n_{\max }} \gamma^{(D-3) n} \times\left[\cos \phi_{1, n}-\cos \left(\frac{2 \pi \gamma^{n} x}{L}+\phi_{1, n}\right)\right]
$$

Morag and Etsion [21] proposed that under a certain contact load the asperity deformation is a result of complete deformation of multiple small-scale asperities. The highest asperity, $n_{n}$, that can support the contact load without being completely crushed was found, and the length scale of this level asperity is referred to the base scale, $l_{n}\left(l_{n}=L / \gamma^{n_{n}}\right)$. Let the random phase $\phi_{1, n}=0$, the cosine expression corresponding to this level of asperity can then be written as follows:

$$
Z_{n}(x)=G^{D-2}(\ln \gamma)^{0.5} l_{n}^{3-D}\left[1-\cos \left(\frac{2 \pi x}{l_{n}}\right)\right]
$$


Figure 4 is a schematic diagram of the geometry for a microcontact between an asperity with level $n_{n}$ and a rigid plane, where $l_{t}$ denotes the truncation diameter; $l_{r}$ denotes the true contact diameter; $R_{n}$ denotes the curvature radius of asperity summit; $\delta_{n}$ represents asperity height; and $\omega_{n}$ represents the asperity interference $\left(0 \leq \omega_{n} \leq \delta_{n}\right)$.

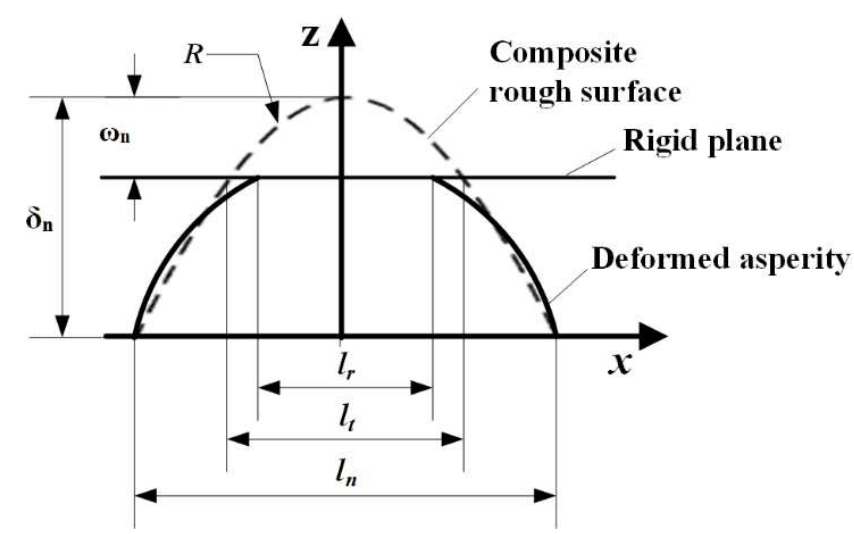

Fig.4 Schematic diagram of geometry of contact asperity with level $n_{n}$.

The asperity height, $\delta_{n}$, is the peak-to-valley amplitude of function $Z_{n}(x)$, which can be written as follows:

$$
\delta_{n}(x)=2 G^{D-2}(\ln \gamma)^{0.5} l_{n}^{3-D}
$$

The interference of asperity, $\omega_{n}$, can be obtained as follows:

$$
\omega_{n}=\delta_{n}-Z_{n}\left(\frac{l^{\prime}}{2}\right)=G^{D-2}(\ln \gamma)^{0.5} l_{n}^{3-D}\left(1+\cos \frac{\pi l_{t}}{l_{n}}\right)
$$

and the radius of curvature, $R_{n}$, as follows:

$$
R_{n}=\left|\left[1+\left(\frac{d Z}{d x}\right)^{2}\right]^{3 / 2} / \frac{d^{2} Z}{d x^{2}}\right|_{x=0}=\frac{l_{n}^{(D-1)}}{4 \pi^{2} G^{(D-2)}(\ln \gamma)^{0.5}}
$$

From the geometric relationships for the asperity, the truncated microcontact area for the asperity is evaluated as follows:

$$
A_{n}=\pi\left(\frac{l_{t}}{2}\right)^{2}=\pi\left[R_{n}^{2}-\left(R_{n}-\omega_{n}\right)^{2}\right] \approx 2 \pi R_{n} \omega_{n}
$$

\subsection{Elastic deformation stage}

Based on the Hertz theory [32], for an asperity in the elastic deformation state, the average contact pressure, $P_{e n}$, the actual microcontact area, $A_{e n}$, and the contact load, $F_{e n}$, are expressed as

$$
\begin{gathered}
P_{e n}=\frac{4 E}{3 \pi} R_{n}^{-0.5} \omega_{n}^{0.5} \\
A_{e n}=\pi R_{n} \omega_{n} \\
F_{e n}=\frac{4 E}{3} R_{n}^{0.5} \omega_{n}^{1.5} \\
7
\end{gathered}
$$


where $E$ denotes composite Young's modulus of the contact material, $1 / E=\left(1-v_{1}^{2}\right) / E_{1}+\left(1-v_{2}^{2}\right) / E_{2}$; $v_{1}$ and $v_{2}$ represent Poisson's ratios of the two contact materials; and $E_{1}$ and $E_{2}$ denote their Young's moduli.

Considering friction between contact surfaces, yield will commence under a critical average pressure [33], $P_{e c n}$, given by

$$
P_{e c n}=1.1 K_{f} \sigma_{y}
$$

where $\sigma_{y}$ denotes the yield strength; and $K_{f}$ denotes the friction correction factor, which is expressed by using the friction factor, $\mu$ as follows [33]:

$$
K_{f}= \begin{cases}1-0.228 \mu & 0 \leq \mu \leq 0.3 \\ 0.932 \exp [-1.58(\mu-0.3)] & 0.3<\mu \leq 0.9\end{cases}
$$

From Eqs. (6), (8) and (11), the critical elastic interference of asperity yielding inception, $\omega_{\text {ecn }}$, can be obtained as follows:

$$
\omega_{e c n}=\left(\frac{33 \pi K_{f} \sigma_{y}}{40 E}\right)^{2} \cdot \frac{l_{n}^{D-1}}{4 \pi^{2} G^{D-2}(\ln \gamma)^{0.5}}
$$

The asperity deformation is elastic until the asperity enters initial yielding. The critical elastic microcontact area is as follows:

$$
A_{e c n}=\pi R_{n} \omega_{e c n}=\pi P\left(\frac{l_{n}^{D-1}}{4 \pi^{2} G^{D-2}(\ln \gamma)^{0.5}}\right)^{2}
$$

where $P=\left(33 \pi K_{f} \sigma_{y} / 40 E\right)^{2}$. For $A_{n} \leq A_{e c n}$, i.e. $\omega_{n} \leq \omega_{e c n}$, the asperity deformation is elastic.

From Eqs. (6), (7), (9) and (10), the contact load acting on the asperity in the elastic deformation regime is obtained as follows:

$$
F_{e n}=\frac{2^{2.5} \pi^{0.5} E G^{D-2}(\ln \gamma)^{0.5}}{3 l_{n}^{D-1}} A_{n}^{1.5}
$$

\subsection{Elastoplastic deformation stage}

In the elastoplastic regime, the solution of the normal average contact pressure for a single asperity is extremely complicated. After loaded, the material hardness, $H$, has no longer a fixed value, but changes instead with the depth of the surface. Thus, for describing better the relationship between the material hardness and the surface depth, a new variable, the ultimate average pressure, $H_{G}(A)$, is introduced as follows [34]:

$$
H_{G}(A)=2.79 K \sigma_{y} A_{e c n}^{-\varepsilon} A_{n}^{\varepsilon}
$$

where $K$ is the material parameter $\left(K=H / \sigma_{y}\right)$. Coefficient $\varepsilon$ is associated with the fractal 
dimension, and is defined as follows [34]:

$$
\varepsilon=\frac{(D-1) \ln K}{\ln 110-(D-1) \ln 2}
$$

Thus, in the elastoplastic deformation stage, the normal contact load for a single asperity is as follows:

$$
F_{e p n}=H_{G}(A) A_{n}=2.79 K \sigma_{y} A_{e c}^{-\varepsilon} A_{n}^{\varepsilon+1}
$$

\subsection{Fully plastic deformation stage}

During the fully plastic contact, the normal average contact pressure for a single asperity equals to its material hardness [32]:

$$
P_{p n}=H
$$

The highest value of the average plastic pressure for a single asperity, $P_{p, \max }$, is as follows [35]:

$$
P_{p, \max }=3 \sigma_{y}
$$

The metal Vickers hardness [32] is given by

$$
H=0.93 P_{p, \max }
$$

From Eq. (19) - (21), the average normal contact pressure is as follows:

$$
P_{p n}=2.79 \sigma_{y}
$$

Kogut and Etsion [12] established using the finite element method (FEM) that when a single asperity starts to reach the plastic deformation stage, the following relationship holds:

$$
\frac{P_{p n}}{\sigma_{y}}=1.61\left(\frac{\omega_{p c n}}{\omega_{e c n}}\right)^{0.117}
$$

From Eqs. (22) and (23), when a single asperity transfers smoothly from the elastoplastic regime to the fully plastic regime, the relationship between the critical plastic deformation and the critical elastic deformation is as follows:

$$
\omega_{p c n}=\left(\frac{279}{161}\right)^{\frac{1}{0.117}} \omega_{e c n} \approx 110 \omega_{e c n}
$$

Substituting Eq. (13) into Eq. (24), the critical plastic deformation is obtained as follows:

$$
\omega_{p c n}=\frac{100 P l_{n}^{D-1}}{4 \pi^{2} G^{D-2}(\ln \gamma)^{0.5}}
$$

When the asperity is completely plastically deformed, the actual microcontact area of a single asperity, $A_{p n}$, is the same as the truncated contact area, namely

$$
\underset{p}{A_{p n}}=A_{n}
$$


Thus, the critical plastic microcontact area is as follows:

$$
A_{p c n}=\frac{25 P l_{n}^{2(D-1)}}{2 \pi^{3} G^{2(D-2)} \ln \gamma}
$$

The contact load for a single asperity can be written as follows:

$$
F_{p n}=K \sigma_{y} A_{n}
$$

\section{Contact model for entire rough surface}

\subsection{Critical levels}

The WM function models the rough surface as comprising asperities with different scale levels. The highest level, $n_{\max }$, corresponds to the shortest length scale of asperity, $l_{\min }\left(l_{\min }=1 / \gamma^{n_{\max }}\right)$. The lowest level, $n_{\min }$, corresponds to the longest length scale of asperity, $l_{\max }\left(l_{\max }=1 / \gamma^{n_{\min }}\right)$, which is determined by the sampling length. Elastic, elastoplastic or completely plastic deformations may be present in these asperities at each length scale. The asperity height of each level is different and the critical interference is also scale-dependent. For an asperity at any level $n$, if the asperity height is smaller than its critical elastic interference under a certain load, then the asperity of this level is only in the elastic deformation regime. In other words, when $\delta_{n} \leq \omega_{e c n}$, combining Eqs. (4) and (13), we can obtain the following:

$$
2 G^{D-2}(\ln \gamma)^{0.5} l_{n}^{3-D} \leq \frac{P \cdot l_{n}^{D-1}}{4 \pi^{2} G^{D-2}(\ln \gamma)^{0.5}}
$$

Thus,

$$
n \leq \frac{1}{2(D-2) \ln \gamma} \ln \left[\frac{L^{2(D-2)} P}{8 \pi^{2} G^{2(D-2)} \ln \gamma}\right]
$$

The critical elastic level is defined as follows:

$$
n_{e c}=\operatorname{int}\left\{\frac{1}{2(D-2) \ln \gamma} \ln \left[\frac{L^{2(D-2)} P}{8 \pi^{2} G^{2(D-2)} \ln \gamma}\right]\right\}
$$

where int \{\} indicates the integer value in the curly brackets.

In the same way, the critical plastic level, $n_{p c}$, is as follows:

$$
n_{p c}=\operatorname{int}\left\{\frac{1}{2(D-2) \ln \gamma} \ln \left[\frac{110 L^{2(D-2)} P}{8 \pi^{2} G^{2(D-2)} \ln \gamma}\right]\right\}
$$

From the above discussion, the asperities of levels $n_{\min } \leq n \leq n_{e c}$ only deform elastically, asperities of levels $n_{e c}<n \leq n_{p c}$ deform elastically or elastoplastically, and asperities of levels $n_{p c}<n \leq n_{\max }$ deform elastically, elastoplastically or fully plastically. 


\subsection{Total contact area and contact load}

Yan and Komvopoulos [19] applied the distribution of islands on earth discovered by Mandelbrot to the three-dimensional surface contact problem, and obtained the size distribution function of the asperity, $n(A)$, as follows:

$$
n(A)=\frac{D-1}{2} A_{L}^{0.5 D-0.5} A^{-0.5 D-0.5}(2<D<3)
$$

where $A_{L}$ is the largest truncated microcontact area. Hence in the YK model, the actual contact area of the rough surface can calculate as follows:

$$
A_{t}=\int_{0}^{A_{L}} n(A) A d A=\frac{D-1}{3-D} A_{L}
$$

Equation (34) shows that the actual contact area of the rough surface, $A_{t}$, is related to the largest truncated microcontact area, $A_{L}$. While in the YK model all the asperities on the surface are modelled by the same distribution, this is not correct for actual surfaces. However, in the present model, each level of asperity has a different base size, which corresponds to a different distribution. From Eq. (3)), in the sample length, the number of asperities at level $n+1$ is $\gamma$ times the number of asperities at level $n$, thus $n_{n+1}(A)=\gamma n_{n}(A)$. Let the truncated area distribution function of the initial level asperity, $n_{\text {min }}$, be $C$ times the total distribution function, then the truncated area distribution function of each level asperity for a rough friction surface is given by

$$
\left\{\begin{array}{c}
n_{\text {min }}(A)=C n(A) \\
n_{\text {min }+1}(A)=\gamma n_{\text {min }}(A) \\
n_{\text {min }+2}(A)=\gamma n_{\text {min }+1}(A) \\
\mathrm{L} \\
n_{\max }(A)=\gamma n_{\max -1}(A)
\end{array}\right.
$$

Thus, the truncated area distribution function of each level of a three-dimensional rough surface can be simplified to

$$
n_{n}(A)=C \gamma^{n-n_{\text {min }}} n(A)\left(n=n_{\text {min }}, n_{\text {min } 1}, \mathrm{~L}, n_{\text {max }}\right)
$$

In the process of simplifying the two-variable WM function to a univariate function, the $\mathrm{x}$-axis and $y$-axis values of the rough surface in the three-dimensional coordinate system are made equal, i.e., the three-dimensional space problem is simplified to the space-plane problem, as shown in Figure 5. Then, the area distribution function of the asperities with each level of the three-dimensional rough surface can be obtained as follows:

$$
n_{n}(A)=C(\sqrt{2} \gamma)^{n-n_{\min }} n(A)\left(n=n_{\min }, n_{\min +1}, \mathrm{~L}, n_{\max }\right)
$$




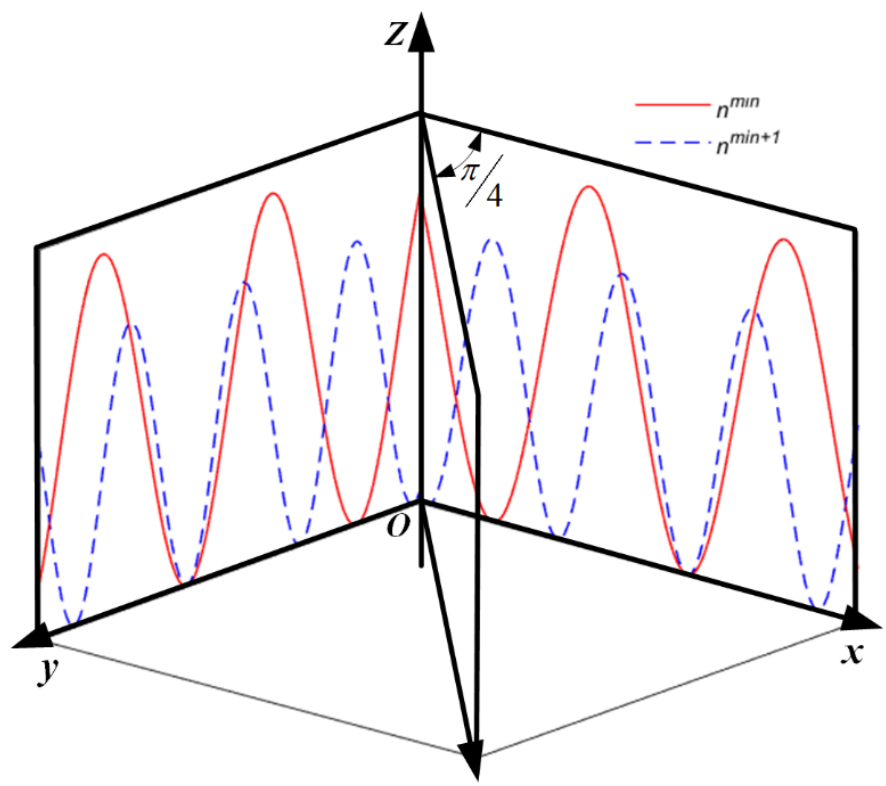

Fig. 5 Schematic diagram of simplified three-dimensional surface model

The actual total contact area of the surface is as follows:

$$
A_{t}=\sum_{n=n_{\min }}^{n_{\max }} \int_{0}^{A_{n L}} n_{n}(A) A d A
$$

where $A_{n L}$ denotes the largest microcontact area of asperities with level $n$, which differs with the level.

Combining Eqs. (34), (37) and (38), we can obtain the following:

$$
C=\frac{A_{L}}{\sum_{n=n_{\text {min }}}^{n_{\text {max }}}\left((\sqrt{2} \gamma)^{n-n_{\text {min }}} A_{n L}\right)}, \quad A_{L}=\max \left\{A_{n L}\right\}
$$

In the process of bringing a fractal friction surface for asperities with level ranging between $n_{\text {min }}$ and $n_{\max }$ into contact with the smooth surface, when the level of asperity, $n$, is $n_{\min } \leq n \leq n_{e c}$, the asperity deforms only elastically. The actual contact area, $A_{r e}$, and the contact load, $F_{r e}$, are calculated as follows:

$$
\begin{gathered}
A_{r e}=\sum_{n=n_{\min }}^{n_{e c}} \int_{0}^{A_{n L}} C(\sqrt{2} \gamma)^{n-n_{\min }} n(A) A d A=\frac{C(D-1)}{3-D} \sum_{n=n_{\text {min }}}^{n_{e c}}(\sqrt{2} \gamma)^{n-n_{\text {min }}} A_{n L} \\
F_{r e}=\sum_{n=n_{\min }}^{n_{e c}} \int_{0}^{A_{n L}} F_{e n} \cdot C(\sqrt{2} \gamma)^{n-n_{\min }} n(A) d A=\frac{C(D-1) 2^{2.5} \pi^{0.5} E G^{D-2}(\ln \gamma)^{0.5}}{3(4-D)} \sum_{n=n_{\min }}^{n_{e c}} \frac{(\sqrt{2} \gamma)^{n-n_{\text {min }}}}{l_{n}^{D-1}} A_{n L}^{1.5}
\end{gathered}
$$

When the level of asperity, $n$, is $n_{e c}<n \leq n_{p c}$, for the case $A_{n L}>A_{e c n}$, elastic deformations or elastoplastic deformations may occur in these asperities. Then the actual contact area, $A_{\text {rep }}$, is composed of the actual area in the elastic deformation, $A_{r e 1}$, and elastoplastic deformation, $A_{r e p 1}$, and is given as follows:

$$
A_{\text {rep }}=A_{\text {re1 }}+A_{\text {rep } 1}
$$




$$
\begin{gathered}
A_{r e 1}=\sum_{n=n_{c c}+1}^{n_{p c}} \int_{0}^{A_{c c n}} C(\sqrt{2} \gamma)^{n-n_{\min }} n(A) A d A=\frac{C(D-1)}{3-D} \sum_{n=n_{e c}+1}^{n_{p c}}(\sqrt{2} \gamma)^{n-n_{\min }} A_{n L}^{0.5 D-0.5} A_{e c n}^{1.5-0.5 D} \\
A_{r e p 1}=\sum_{n=n_{c c}+1}^{n_{p c c}} \int_{A_{c c n}}^{A_{n L}} C(\sqrt{2} \gamma)^{n-n_{\min }} n(A) A d A=\frac{C(D-1)}{3-D} \sum_{n=n_{c c}+1}^{n_{p c c}}(\sqrt{2} \gamma)^{n-n_{\min }} A_{n L}^{0.5 D-0.5}\left(A_{n L}^{1.5-0.5 D}-A_{e c n}^{1.5-0.5 D}\right)
\end{gathered}
$$

The contact load, $F_{\text {rep }}$, can be evaluated as follows:

$$
\begin{gathered}
F_{r e p}=F_{r e 1}+F_{r e p 1} \\
F_{r e 1}=\sum_{n=n_{e c}+1}^{n_{p c}} \int_{0}^{A_{c e n}} F_{e n} C(\sqrt{2} \gamma)^{n-n_{\min }} n(A) d A \\
=\frac{C(D-1) 2^{2.5} \pi^{0.5} E G^{D-2}(\ln \gamma)^{0.5}}{3(4-D)} \sum_{n=n_{e c}+1}^{n_{p c}} \frac{(\sqrt{2} \gamma)^{n-n_{\min }}}{l_{n}^{D-1}} A_{n L}^{0.5 D-0.5} A_{e c n}^{2-0.5 D} \\
F_{r e p 1}=\sum_{n=n_{c c}+1}^{n_{p c}} \int_{A_{c c n}}^{A_{n L}} F_{e p n} C(\sqrt{2} \gamma)^{n-n_{\min }} n(A) d A \\
=\frac{2.79 C(D-1) K \sigma_{y}}{2 \varepsilon-D+3} \sum_{n=n_{e c}+1}^{n_{p c}}(\sqrt{2} \gamma)^{n-n_{\min }}\left(A_{n L}^{\varepsilon+1} A_{e c n}^{-\varepsilon}-A_{n L}^{0.5 D-0.5} A_{e c n}^{1.5-0.5 D}\right)
\end{gathered}
$$

When the level of asperity $n$ is $n_{p c}<n \leq n_{\max }$, for the case $A_{n L}>A_{p c n}$, elastic deformations, elastoplastic deformations or fully plastic deformations may occur in these asperities. Then the actual contact area, $A_{r p}$, is composed of the actual contact area in the elastic regime, $A_{r e 2}$, the elastoplastic regime, $A_{r e p 2}$, and fully plastic regime, $A_{r p 2}$, as follows:

$$
\begin{aligned}
& A_{r p}=A_{r e 2}+A_{r e p 2}+A_{r p 2} \\
& A_{r e 2}=\sum_{n=n_{p c}+1}^{n_{m a x}} \int_{0}^{A_{c e n}} C(\sqrt{2} \gamma)^{n-n_{\min }} n(A) A d A=\frac{C(D-1)}{3-D} \sum_{n=n_{p c}+1}^{n_{m a x}}(\sqrt{2} \gamma)^{n-n_{\min }} A_{n L}^{0.5 D-0.5} A_{e c n}^{1.5-0.5 D} \\
& A_{\text {rep } 2}=\sum_{n=n_{p c}+1}^{n_{\text {max }}} \int_{A_{\text {ecn }}}^{A_{p e n}} C(\sqrt{2} \gamma)^{n-n_{\min }} n(A) A d A=\frac{C(D-1)}{3-D} \sum_{n=n_{p c}+1}^{n_{m a x}}(\sqrt{2} \gamma)^{n-n_{\min }} A_{n L}^{0.5 D-0.5}\left(A_{p c n}^{1.5-0.5 D}-A_{e c n}^{1.5-0.5 D}\right) \\
& A_{r p 2}=\sum_{n=n_{p c}+1}^{n_{m a x}} \int_{A_{p c n}}^{A_{n L}} C(\sqrt{2} \gamma)^{n-n_{\min }} n(A) A d A=\frac{C(D-1)}{3-D} \sum_{n=n_{p c}+1}^{n_{m a x}}(\sqrt{2} \gamma)^{n-n_{\min }} A_{n L}^{0.5 D-0.5}\left(A_{n L}^{1.5-0.5 D}-A_{p c n}^{1.5-0.5 D}\right)
\end{aligned}
$$

The contact load, $F_{r p}$, can be evaluated as follows:

$$
\begin{gathered}
F_{r p}=F_{r e 2}+F_{r e p 2}+F_{r p 2} \\
F_{r e 2}=\sum_{n=n_{p c}+1}^{n_{m a x}} \int_{0}^{A_{c n}} F_{e n} C(\sqrt{2} \gamma)^{n-n_{\min }} n(A) d A \\
=\frac{C(D-1) 2^{2.5} \pi^{0.5} E G^{D-2}(\ln \gamma)^{0.5}}{3(4-D)} \sum_{n=n_{p c}+1}^{n_{m a x}} \frac{(\sqrt{2} \gamma)^{n-n_{m i n}}}{l_{n}^{D-1}} A_{n L}^{0.5 D-0.5} A_{e c n}^{2-0.5 D} \\
F_{r e p 2}=\sum_{n=n_{p c}+1}^{n_{m a x}} \int_{A_{c c n}}^{A_{p e n}} F_{e p n} C(\sqrt{2} \gamma)^{n-n_{\min }} n(A) d A \\
=\frac{2.79 C(D-1) K \sigma_{y}}{2 \varepsilon-D+3} \sum_{n=n_{p c}+1}^{n_{m a x}}(\sqrt{2} \gamma)^{n-n_{\min }} A_{n L}^{0.5 D-0.5} A_{e c n}^{-\varepsilon}\left(A_{p c n}^{\varepsilon-0.5 D+1.5}-A_{e c n}^{\varepsilon-0.5 D+1.5}\right)
\end{gathered}
$$




$$
\begin{aligned}
F_{r p 2} & =\sum_{n=n_{p c}+1}^{n_{m a x}} \int_{A_{p c n}}^{A_{n L}} F_{p n} C(\sqrt{2} \gamma)^{n-n_{\min }} n(A) d A \\
& =\frac{C(D-1) K \sigma_{y}}{3-D} \sum_{n=n_{p c}+1}^{n_{m a x}}(\sqrt{2} \gamma)^{n-n_{\min }} A_{n L}^{0.5 D-0.5}\left(A_{n L}^{1.5-0.5 D}-A_{p c n}^{1.5-0.5 D}\right)
\end{aligned}
$$

Thus, the actual total contact area for all asperity levels is as follows:

$$
\begin{aligned}
A_{r t}= & A_{r e}+A_{r e p}+A_{r p} \\
= & \sum_{n=n_{\min }}^{n_{c e}} \int_{0}^{A_{n L}} C(\sqrt{2} \gamma)^{n-n_{\min }} n(A) A d A+\sum_{n=n_{c c}+1}^{n_{m a x}} \int_{0}^{A_{c c n}} C(\sqrt{2} \gamma)^{n-n_{\min }} n(A) A d A \\
& +\sum_{n=n_{c c}+1}^{n_{p c}} \int_{A_{c c n}}^{A_{n L}} C(\sqrt{2} \gamma)^{n-n_{\text {min }}} n(A) A d A+\sum_{n=n_{p c}+1}^{n_{m a x}} \int_{A_{c c n}}^{A_{p c c}} C(\sqrt{2} \gamma)^{n-n_{\text {min }}} n(A) A d A \\
& +\sum_{n=n_{p c}+1}^{n_{m a x}} \int_{A_{p c n}}^{A_{n L}} C(\sqrt{2} \gamma)^{n-n_{\min }} n(A) A d A
\end{aligned}
$$

and the total normal contact load for all asperity levels is as follows:

$$
\begin{aligned}
F_{r t}= & F_{r e}+F_{r e p}+F_{r p} \\
= & \sum_{n=n_{\min }}^{n_{c e c}} \int_{0 n}^{A_{n L}} F_{e n} C(\sqrt{2} \gamma)^{n-n_{\min }} n(A) d A+\sum_{n=n_{e c}+1}^{n_{m a x}} \int_{0}^{A_{c e n}} F_{e n} C(\sqrt{2} \gamma)^{n-n_{\min }} n(A) d A \\
& +\sum_{n=n_{c e}+1}^{n_{p c}} \int_{A_{c e n}}^{A_{n L}} F_{e p n} C(\sqrt{2} \gamma)^{n-n_{\min }} n(A) d A+\sum_{n=n_{p c}+1}^{n_{m a x}} \int_{A_{c e n}}^{A_{p c n}} F_{e p n} C(\sqrt{2} \gamma)^{n-n_{\min }} n(A) d A \\
& +\sum_{n=n_{p c}+1}^{n_{m a x}} \int_{A_{p e n}}^{A_{n L}} F_{p n} C(\sqrt{2} \gamma)^{n-n_{\min }} n(A) d A
\end{aligned}
$$

In order to facilitate numerical analysis, normalization of variables was carried out. The nondimensional total actual contact area is denoted by $A_{r t}^{*}, A_{r t}^{*}=A_{r t} / A_{a}$, where $A_{a}$ represents the apparent area of rough friction surface defined as the square of sampling length, $L$, i.e., $A_{a}=L^{2}$. The nondimensional total contact load is denoted by $F_{r t}^{*}, F_{r t}^{*}=F_{r t} /\left(A_{a} E\right)$.

\section{Results and discussion}

Table 1 lists the geometric and material parameters of the rough surface assumed in numerical simulations of the proposed model.

Table 1. Material and geometric parameters used in calculations

\begin{tabular}{ll}
\hline Parameter & Value \\
\hline Nominal contact area of friction surface $A_{a}\left[\mathrm{~m}^{2}\right]$ & $1.0 \times 10^{-6}$ \\
Poisson's ratios $v_{1}$ and $v_{2}[-]$ & 0.25 \\
Elastic moduli $E_{1}$ and $E_{2}[\mathrm{~Pa}]$ & $2.06 \times 10^{11}$ \\
Yield strength $\sigma_{y}[\mathrm{~Pa}]$ & $3.45 \times 10^{8}$
\end{tabular}




\begin{tabular}{ll} 
Hardness $H\left[\mathrm{~N} / \mathrm{m}^{2}\right]$ & $5 \times 10^{8}$ \\
Friction coefficient $\mu \quad[-]$ & 0.1 \\
Shortest truncated length $L_{h}[\mathrm{~m}]$ & $1.0 \times 10^{-13}$ \\
\hline
\end{tabular}

Figure 6 shows the relationship between different fractal parameters ( $D$ and $G$ ) and the critical level of asperity for a certain sampling length of the rough surface. It can be seen that at a certain fractal dimension, $D$, the critical scale level of asperity is negatively correlated with the fractal roughness, $G$. However, the critical scale level of asperity is positively correlated with the fractal dimension, $D$, for a certain fractal roughness, $G$. This is because the fractal parameters are related to the asperity height. For the same contact load, the higher the asperity height, the fewer the asperity levels for the rough surface that remain only in the elastic regime, hence the lower the critical level value. For rough friction surfaces with the same fractal parameters, the critical scale level of elastic deformation is smaller than that of fully plastic deformation. All the asperities below the elastic critical level surface are exclusively in the elastic regime, all the asperities between the elastic and the fully plastic critical level surfaces may be in the elastic or elastoplastic regime, and all the asperities above the fully plastic critical level surfaces may be in any regime including elastic, elastoplastic or fully plastic.

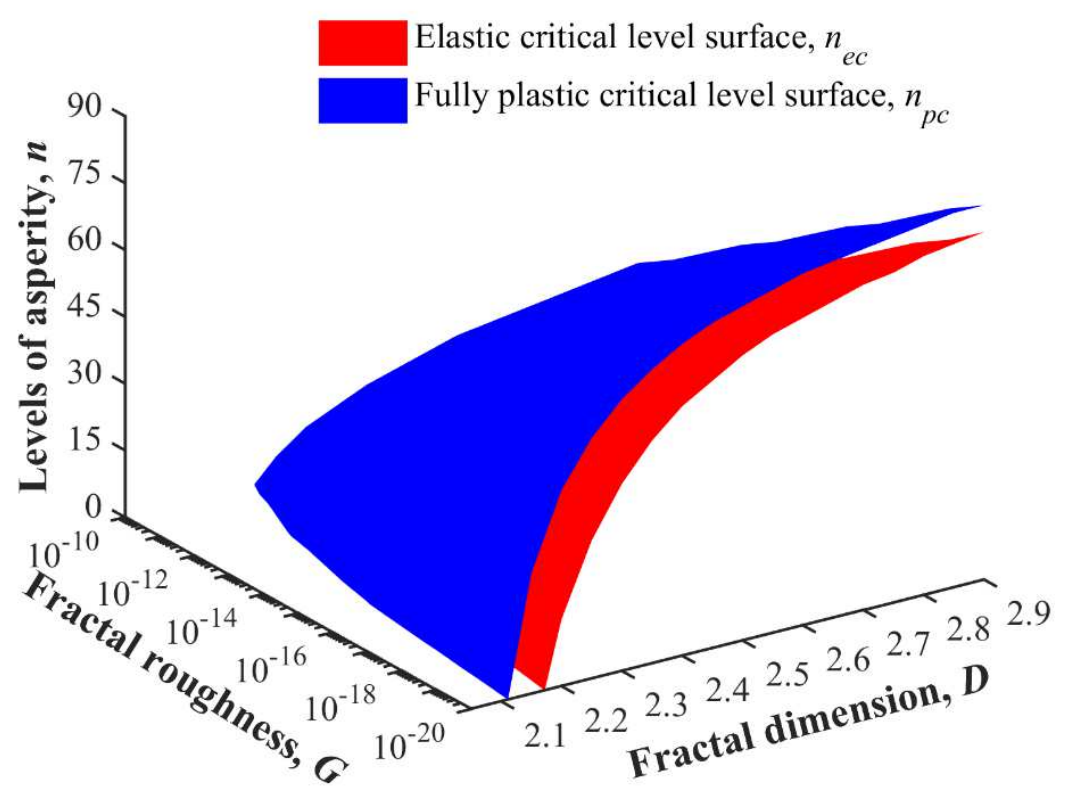

Fig. 6 Relationship among fractal roughness, fractal dimension and critical asperity levels.

In this section, in order to investigate the variation of the actual contact area with the contact load applied to the rough surface, the model is simplified. In the process of contact between the rough friction surface and the smooth rigid surface, as the load increases, we assume that the deformation of each level of asperity on the rough surface is proportional to its own height, i.e., $\omega_{n}=N \delta_{n}, 0 \leq N \leq 1$. For fractal dimension $D=2.8$, sample length $L=1.0 \times 10^{-3} \mathrm{~m}$, fractal roughness $G=1.36 \times 10^{-15} \mathrm{~m}$, and coefficient of 
friction $\mu=0.1$, we can obtain the range of asperity levels from $n_{\min }=17$ to $n_{\max }=56$. The elastic critical level, $n_{e c}$, and the fully plastic critical level, $n_{p c}$, are 47 and 54 , respectively. Thus, all the asperities with scale levels, $n$, from 17 to 47 are only in elastic contact, all the asperities with scale levels, $n$, from 48 to 54 may be in elastic deformation or elastoplastic deformation, and all the asperities with scale levels, $n$, from 55 to 56 may be in elastic deformation, elastoplastic deformation or fully plastic deformation. The relationship between the dimensionless contact load, $F_{r t}^{*}$, and the dimensionless actual contact area, $A_{r t}^{*}$, during the contact process is shown in Figure 7 for asperity levels ranging from 17 to 56 . The results demonstrate that the nondimensional total actual contact area increases with the increase in the nondimensional total normal contact load. $F_{r t}^{*}$ is in a nonlinear relationship to $A_{r t}^{*}$ in the initial stage, but with increasing $F_{r t}^{*}$ this relationship becomes nearly linear. This happens because the proportion of asperities in the elastic regime and elastoplastic regime gradually decreases during the contact process.

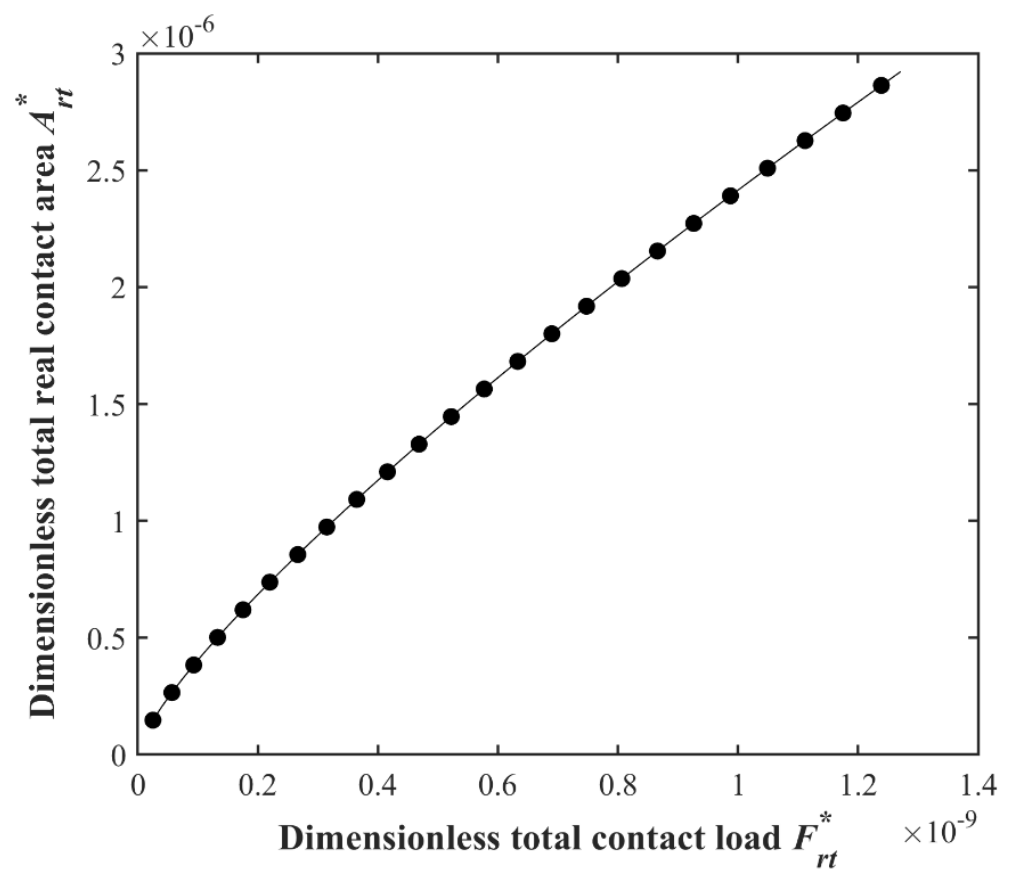

Fig. 7. Total nondimensional actual contact area vs. nondimensional contact load

$$
\text { for } n_{\min }=17 \text { and } n_{\max }=56
$$

Figure 8 shows the plot between the critical actual microcontact area and the scale level of asperity for $D=2.8$ and $G=1.36 \times 10^{-14} \mathrm{~m}$ in a logarithmic scale. It can be seen that the critical actual microcontact area decreases with the increase in the asperity scale level. This is because as the asperity level increases, the geometrical dimensions, including the asperity height and the radius of curvature, decrease. This indicates that the critical elastic actual microcontact area and the critical plastic actual microcontact area for the rough friction surface are both scale dependent. For a fixed asperity scale level, $n$, the critical plastic microcontact area is larger than the critical elastic microcontact area. Under contact load, a single asperity initially deforms elastically. With increasing normal load, the single asperity will transit to the 
elastoplastic regime and then to the fully plastic regime. These results are consistent with the classical Hertz contact theory [32], but not with the YK model.

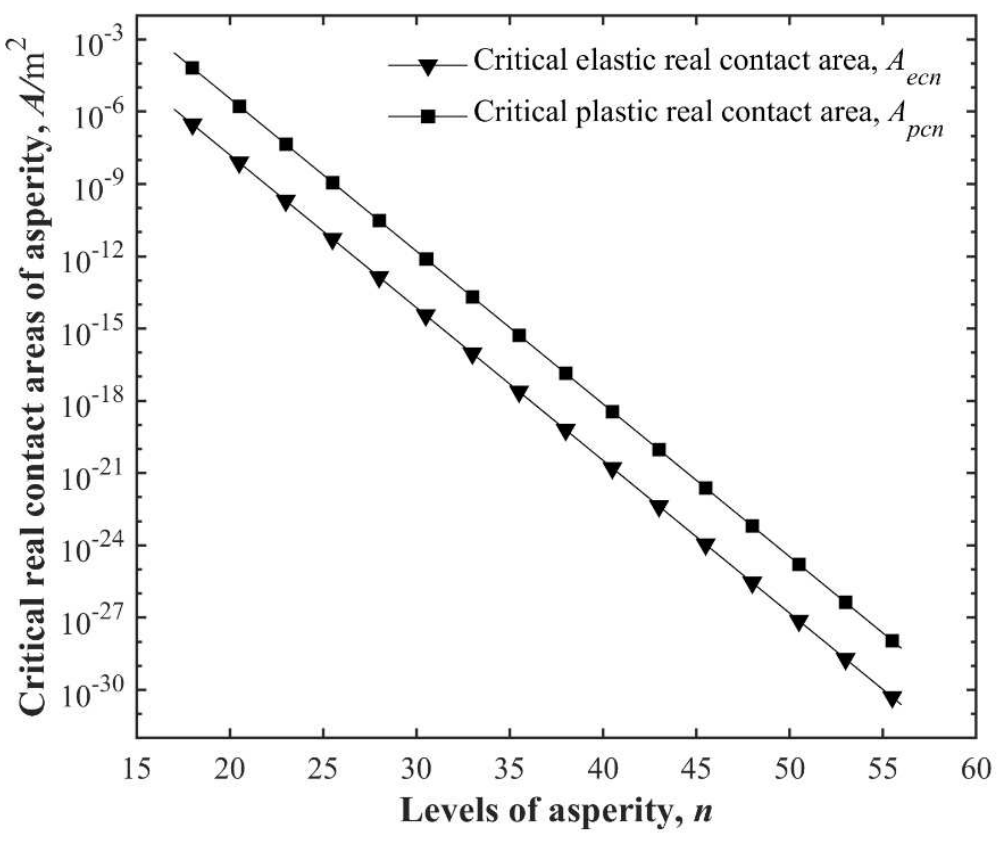

Fig. 8 Critical actual contact areas vs. levels of asperity

Figure 9 shows, the ratios of various level asperity actual microcontact area to the total actual contact area, $A_{r n} / A_{r t}$, for a friction surface with for $D=2.8$ and $G=1.36 \times 10^{-14}$. The figure reveals that the actual microcontact area for each level of asperity is scale dependent. Under the same conditions, the lowest level of asperities on the rough surface has the highest value of $A_{r n} / A_{r t}$. As the asperity level increases, the $A_{r n} / A_{r t}$ value decreases, but the rate of decrease becomes slower. For example, for the rough surface with $L=0.01 \mathrm{~m}$ and $\mu=0.1$, the $A_{r n} / A_{r t}$ value of the asperity decreases rapidly before level $n=40$. This is because in the simulations, the asperity level range is from $n_{\min }=11$ and $n_{\max }=62$, the elastic critical level is 47 and the fully plastic critical level is 54 . The levels, $n$, from 11 to 47 are for asperities in elastic contact during the contact process. However, various values of friction coefficients have no effect on the proportion of actual contact areas at each level of asperities on the rough surface. For the same level of asperities, the value of $A_{r n} / A_{r t}$ increases as the sampling length decreases. Because the sampling length determines the range of levels of the asperity on the rough surface, the reduction in the sampling length leads to a smaller range of the asperity levels. 


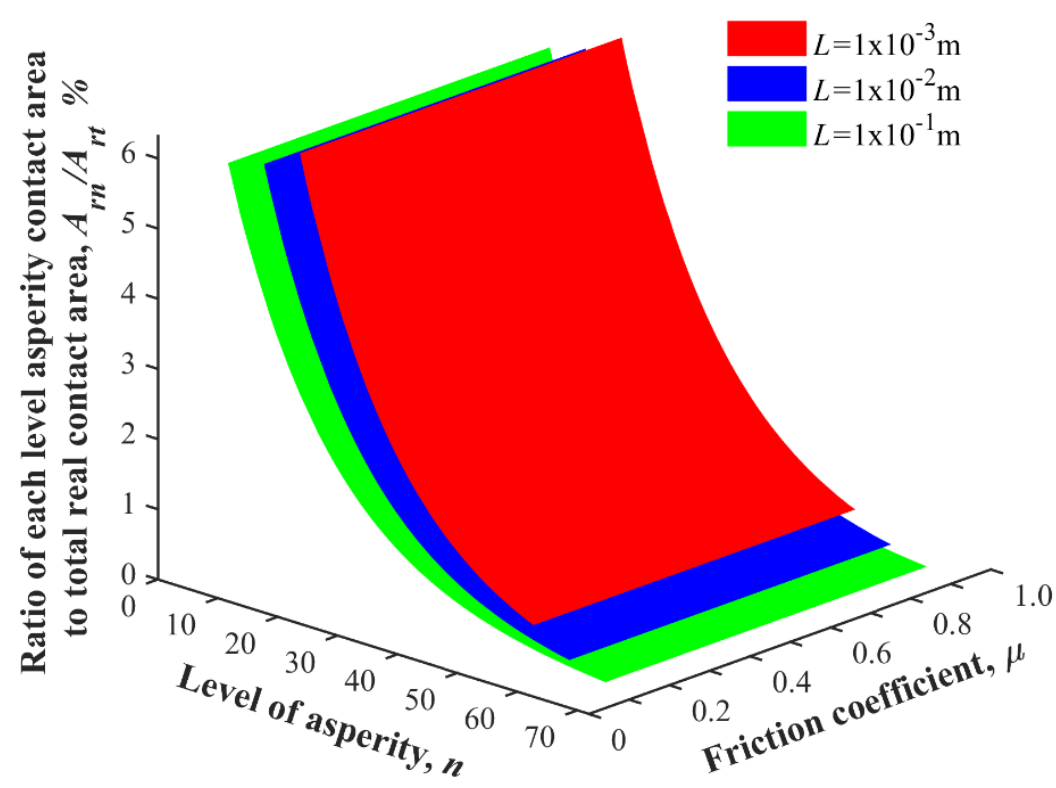

Fig. 9 Ratio of each level asperity microcontact area to total actual contact area.

Ausloos et al. [27] demonstrated that during the process of surface wear, plastic contact has a more significant influence on surface wear than elastic contact. That is, for the total actual contact area, a larger proportion of the elastic contact area is beneficial for reducing wear. In this model, $A_{r e t} / A_{r t}$ is the ratio of the total elastic contact area of asperities on the rough surface whose value is equal to the total elastic microcontact area generated by all asperities of the rough surface with elastic deformation to the total actual contact area. For a fixed value of $G=1.36 \times 10^{-14} \mathrm{~m}$, the relationship between $A_{r e t} / A_{r t}$ and the dimensionless contact area, $A_{r t}^{*}$, and fractal dimension, $D$, is given in Figure 10. It can be seen from the figure that with the increase in contact load, $A_{r t}^{*}$ of the surface gradually increases and $A_{r e t} / A_{r t}$ decreases. On the other hand, as $D$ of the rough surface increases, $A_{r e t} / A_{r t}$ decreases more slowly. This is because, with the increasing normal contact load, asperities on the rough surface gradually transfer from elastic deformations to elastoplastic deformations to fully plastic deformations. This causes an increase of the contact area produced by elastoplastic deformations and fully plastic deformations on the surface. Thus, the value of $A_{r e t} / A_{r t}$ reduces. Under the same conditions, $n_{e c}$ will increase as $D$ of the rough surface increases. Then, the range of levels of asperities with elastic deformations on rough surface is larger than that with elastoplastic deformations or fully plastic deformations. Thus, as the load increases, $A_{r e t} / A_{r t}$ decreases more slowly. 


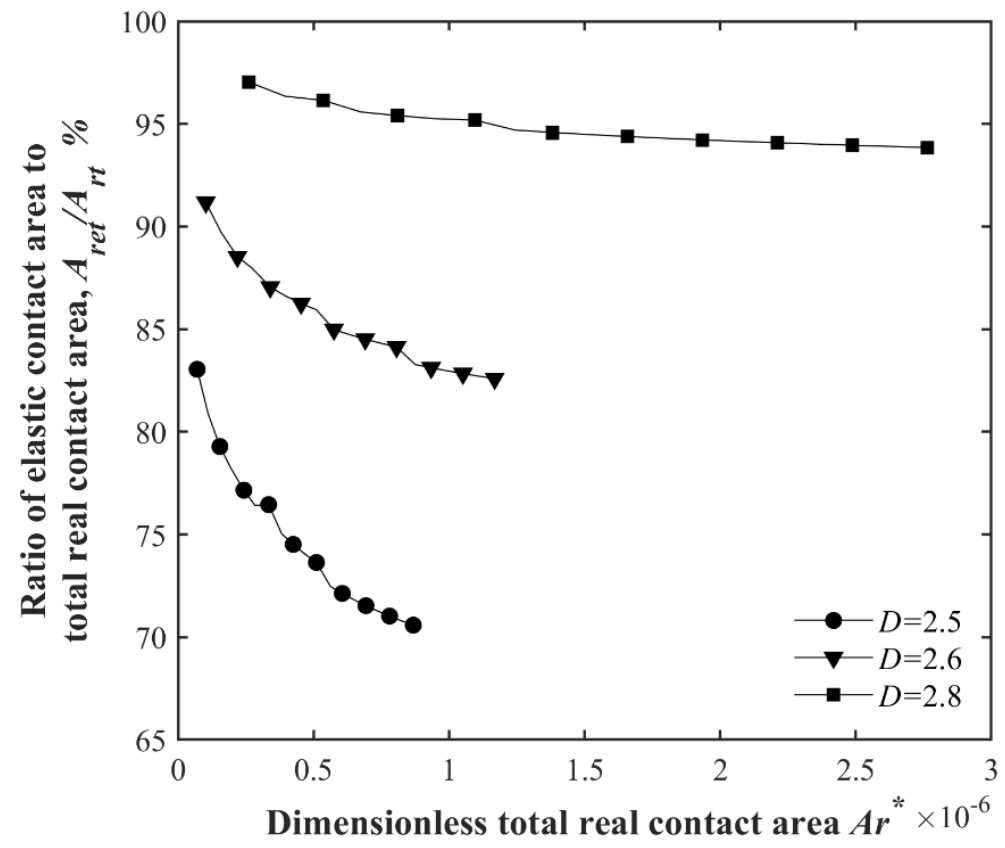

Fig. 10 Ratio of elastic contact area to total actual contact area.

Figure 11 shows the plots of dimensionless total actual contact area as a function of the nondimensional total contact load for the fixed value $G=1.36 \times 10^{-14} \mathrm{~m}$, the asperity levels ranging from 17 to 56, and varying fractal dimension, $D$. With the increase in normal contact load, the increase in $D$ makes the total actual contact area grow faster. Moreover, for a fixed value of $G$, it can be seen that under a particular load value, increasing the value of $D$ raises the total actual contact area. This is because a greater value of $D$ corresponds to a smoother surface. Therefore, the actual contact area is greater for the surface which has a larger value of $D$ under a certain normal contact load.

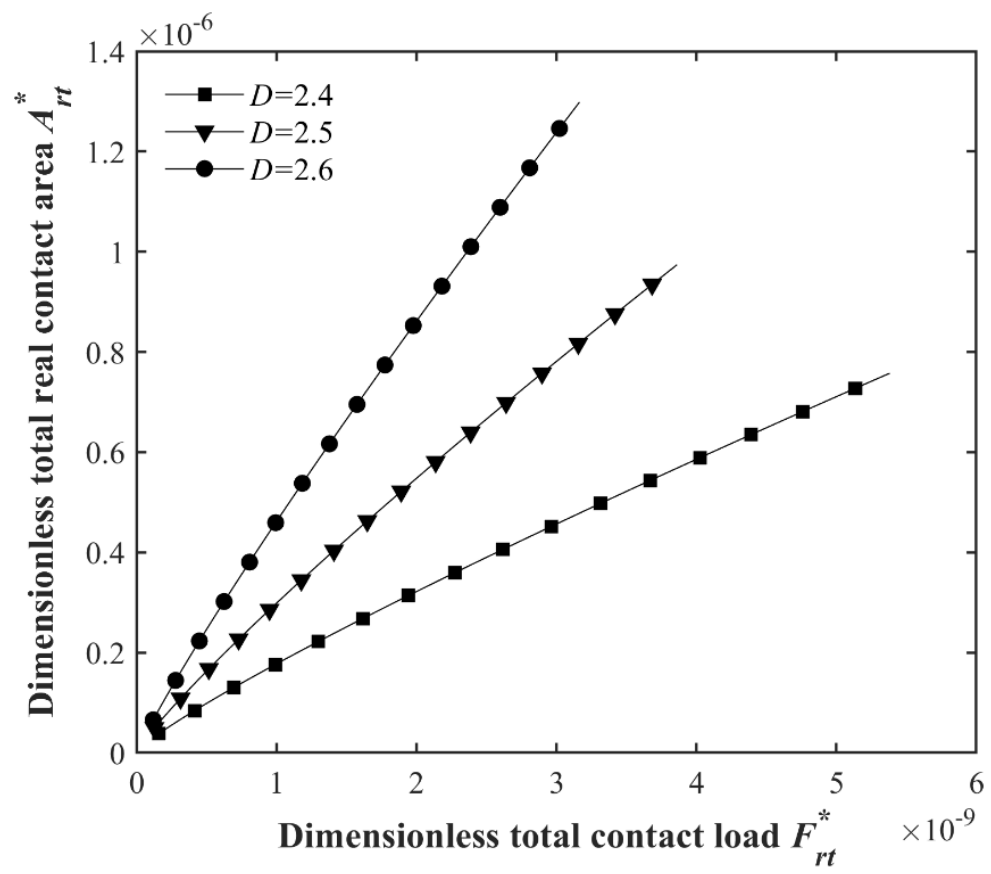

Fig. 11 Relationships among nondimensional total contact area, fractal dimension and nondimensional total contact load for $n_{\min }=17$ and $n_{\max }=56$. 
To verify the proposed model, its results are compared to the results produced by the YK fractal model [19], the FEM numerical model [4], and the experimental data given by Kucharski et al. [36]. Kucharski et al. [36] carried out measurements of the contact area and contact load using carbon steel $\left(0.45 \%\right.$ carbon) specimens with Young's modulus $E=2.0 \times 10^{11} \mathrm{~Pa}$, Poisson's ratio $v=0.3$, hardness $H=1.12 \times 10^{9} \mathrm{~Pa}$, and tensile yield strength $\sigma_{y}=4.0 \times 10^{8} \mathrm{~Pa}$. In the present silumation, the fractal parameters are chosen as $D=2.3$ and $G=1.36 \times 10^{-11} \mathrm{~m}$. A comparison of the proposed model with the YK model [19], the FEM model [4] and the experimental results [36] for the nondimensional total actual contact area variation with nondimensional total contact load is shown in Fig. 12. It can be seen that all models exhibit the same trend as the experimental results, where increasing the normal load increases the total actual contact area. In the initial stage, the FEM model fits the experimental results better, while the proposed model shows some deviation. With the increase in load $F_{r t}^{*}$, the predicted results of the proposed model fit the experimental results best, while the numerical FEM model comes second. Moreover, the growth trend of our model is also closer to the experimental data. On the other hand, the analytical solution of the YK fractal model differs significantly from the experimental data. This is because the YK model ignores the influence of the scale level of the asperities on the rough surface and assumes elastoplastic contact.

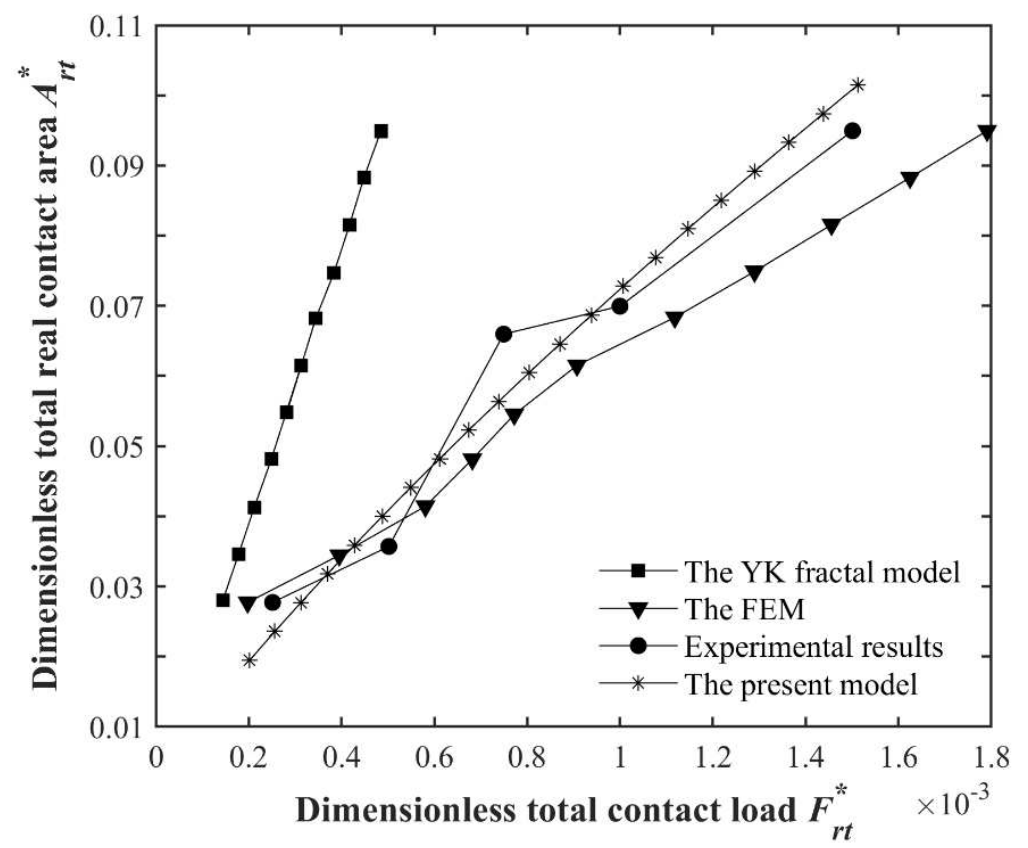

Fig. 1 Comparison of proposed fractal model, YK fractal model, and FEM model with experimental results.

\section{Conclusions}

In this paper, a contact prediction model for three-dimensional friction rough surface with multiplescale asperities has been established based on the fractal and Hertz theories. We have deduced the 
microcontact fractal model of asperities at multiple-scale levels and revised the size distribution function of them. The relationships among levels of asperity, fractal parameters, total contact area, and total normal contact load were given by numerical simulations. The specific conclusions are given as follows:

(1) The critical interferences and the critical microcontact areas for a single asperity are all related to scale. The microcontact area of critical elastic deformation is smaller than that of critical fully plastic deformation for each level of asperities. With an increase in contact loading, a transition from elastic to elastoplastic to fully plastic deformation occurs. This is consistent with the traditional Hertz contact theory.

(2) Based on the modified truncation size distribution function, the actual microcontact area of each level asperity was calculated. The elastic contact area ratio reduces gradually with the raising of the contact area. Moreover, it is positively correlated with the fractal dimension.

(3) As an increase in fractal dimension, the contact load on the rough surface is almost linearly related to the contact area. In contrast to the YK fractal model and the FEM model, the results of the proposed model are close to the experimental results.

The present model retains the concept of multiple-scale asperities. Friction, wear and adhesion on rough surfaces can be further studied in the context of the present theory.

\section{Acknowledgments}

The authors would like to acknowledge the support of the National Natural Science Foundation of China (Grant No. 51875412) and the Shanghai Natural Science Foundation (Grant No. 18ZR1441000).

\section{Declarations}

\subsection{Funding}

The research leading to these results received funding from the National Natural Science Foundation of China under Grant Agreement No. 51875412 and the Shanghai Natural Science Foundation under Grant Agreement No. 18ZR1441000.

\subsection{Availability of data and material}

Not applicable

\subsection{Code availability}

Not applicable

\subsection{Ethics approval}

This article does not contain any studies on humans or animals performed by any of the authors. In 
this experiment, we did not collect any samples of human and animal material.

\subsection{Consent to participate}

Not applicable

\subsection{Consent for publication}

Not applicable

\section{References}

[1] Zhang, C., Ren, W.: Modeling of 3D surface morphologies for predicting the mechanical contact behaviors and associated electrical contact resistance. Tribol. Lett. 69(1), 20 (2021)

[2] Tsukanov, I.Y.: An extended asymptotic analysis for elastic contact of three-dimensional wavy surfaces. Tribol. Lett. 67(4), 107 (2019)

[3] Chatterjee, A., Bowling, A.: Modelling three-dimensional surface-to-surface rigid contact and impact. Multibody Syst. Dyn. 46(1), 1-40 (2019)

[4] Sahoo, P., Ghosh, N.: Finite element contact analysis of fractal surfaces. J. Phys. D. Appl. Phys. 40(14), 4245 (2007)

[5] Yeo, C., Katta, R., Polycarpou, A.: Improved elastic contactmodel accounting for asperity and bulk substrate deformation. Tribol. Lett. 35, 191-203 (2009)

[6] Mu, X., Sun, Q., Xu, J., Chai, Z., Sun, W., Zhao, B.: Feasibility analysis of the replacement of the actual machining surface by a 3D numerical simulation rough surface. Int. J. Mech. Sci.

150, 135-144 (2018)

[7] Jana, T., Mitra, A., Sahoo, P.: Contact behavior of functionally graded fractal surface. P. I. Mech. Eng. L-J. Mat. 234(10), 1355-1374 (2020)

[8] Kogut, L., Jackson, R.L.: A comparison of contact modeling utilizing statistical and fractal approaches. J. Tribol. 128(1), 213-217 (2006)

[9] Greenwood, J.A., Williamson, J.B.P.: Contact of nominally flat surfaces. P roy. Soc. A-Math. Phy. 295(1442), 300-319 (1966)

[10] Chang, W.R., Etsion, I., Bogy, D.B.: An elastic-plastic model for the contact of rough surfaces. J. Tribol. 109(2), 257-263 (1987)

[11] Zhao, Y., Maietta, D.M., Chang, L.: An asperity microcontact model incorporating the transition from elastic deformation to fully plastic flow. J. Tribol. 122(1), 86-93 (2000)

[12] Kogut, L., Etsion, I.: Elastic-plastic contact analysis of a sphere and a rigid flat. Int. J. Appl. Mech. 69(5), 657-662 (2002)

[13] Cohen, D., Kligerman, Y., Etsion, I.: A model for contact and static friction of nominally flat rough surfaces under full stick contact condition. J. Tribol. 130(3), 031401-031409 (2008)

[14] Xu, Y., Jackson, R.L., Marghitu, D.B.: Statistical model of nearly complete elastic rough surface contact. Int. J. Solids Struct. 51(5), 1075-1088 (2014) 
[15] Song, H., Vakis, A.I., Liu, X., Giessen, E.: Statistical model of rough surface contact accounting for size-dependent plasticity and asperity interaction. J. Mech. Phys. Solids. 106, 1-14 (2017)

[16] Persson, B.N.J.: On the Fractal Dimension of Rough Surfaces. Tribol. Lett. 54, 99-106 (2014)

[17] Majumdar, A., Bhushan, B.: Fractal model of elastic-plastic contact between rough surfaces. J. Tribol. 113(1), 1-11 (1991)

[18] Wei, L., Liu, Q., Zhang, P.: Sliding friction surface contact mechanics model based on fractal theory. J. Mech. En. 48(17), 106-113 (2012)

[19] Yan, W., Komvopoulos, K.: Contact analysis of elastic-plastic fractal surfaces. J. Appl. Phys. 84(7), 3617-3624 (1998)

[20] Liu, P., Zhao, H., Huang, K., Chen, Q.: Research on normal contact stiffness of rough surface considering friction based on fractal theory. Appl. Surf. Sci. 349, 43-48 (2015)

[21] Morag, Y., Etsion, I.: Resolving the contradiction of asperities plastic to elastic mode transition in current contact models of fractal rough surfaces. Wear. 262(5-6), 624-629 (2007)

[22] Liou, J.L., Lin, J. F.: A modified fractal microcontact model developed for asperity heights with variable morphology parameters. Wear. 268(1), 133-144 (2010)

[23] Jackson, R.L., Streator, J.L.: A multi-scale model for contact between rough surfaces. Wear. 261(11), 1337-1347 (2006)

[24] Miao, X., Huang, X.: A complete contact model of a fractal rough surface. Wear. 309(1-2), 146-151 (2014)

[25] Pan, W., Li, X., Wang, L., Mu, J., Yang, Z.: A loading fractal prediction model developed for dry-friction rough joint surfaces considering elastic-plastic contact. Acta Mech. 229(5), 2149-2162 (2018)

[26] Wang, H., Jia, P., Wang, L., Yun, F., Liu, M.: Research on the loading-unloading fractal contact model between two three-dimensional spherical rough surfaces with regard to friction. Acta Mech. 231(10), 4397-4413 (2020)

[27] Ausloos, M., Berman, D.H.: A multivariate Weierstrass-Mandelbrot function. P. Roy. Soc. AMath. Phy. 400(1819), 331-350 (1985)

[28] Hao, Q., Yi, S., Yan, W., Xin, Z.: A fractal model of acoustic emission signals in sliding friction. Tribol. Lett. 67(1), 31 (2019)

[29] Song, Z., Komvopoulos, K.: Contact mechanics analysis of oscillatory sliding of a rigid fractal surface against an elastic-plastic half-space. Philos. Mag. 94(28), 3215-3233 (2014)

[30] Bora, C.K., Flater, E.E., Streeta, M.D., Redmondb, J.M., Starrb, M.J., Carpickaand, R.W., Plesha, M.E.: Multiscale roughness and modeling of MEMS interfaces. Tribol. Lett. 19(1), 37-48 (2005)

[31] Majumdar, A., Tien, C.L.: Fractal characterization and simulation of rough surfaces. Wear. 136(2), 313-327 (1990)

[32] Johnson, K.L.: Contact Mechanics. Cambridge University Press, Cambridge (1985) 
[33] Wang, S., Komvopoulos, K.: A fractal theory of the interfacial temperature distribution in the slow sliding regime: part II - multiple domains, elastoplastic contacts and applications. J.

Tribol. 116(4), 824-832 (1994)

[34] Tian, H., Zhong, X., Zhao, C., Zhao, X., Fang, Z., Liu, F., Zhu, D., Lin, W., Yan, H.: One loading model of joint interface considering elastoplastic and variation of hardness with surface depth. J. Mech. En. 000(005), 90-104 (2015)

[35] TABOR, D.: The Hardness of Metals. Oxford University Press, Oxford, UK (1951)

[36] Kucharski, S., Klimczak, T., Polijaniuk, A., Kaczmarek, J.: Finite-elements model for the contact of rough surfaces. Wear. 177(1), 1-13 (1994) 


\section{Figures}

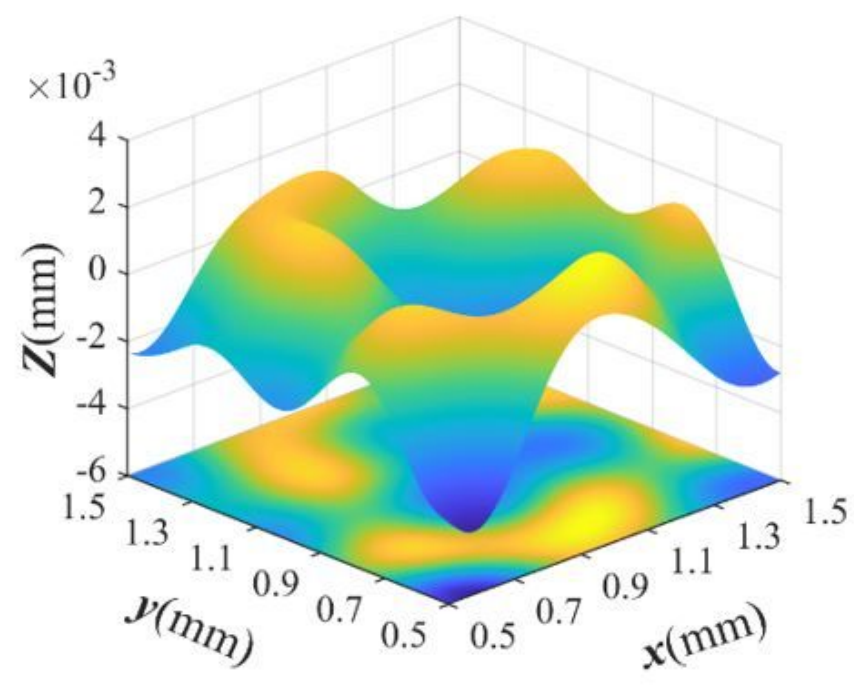

(a) $\mathrm{n}_{\max }=2$

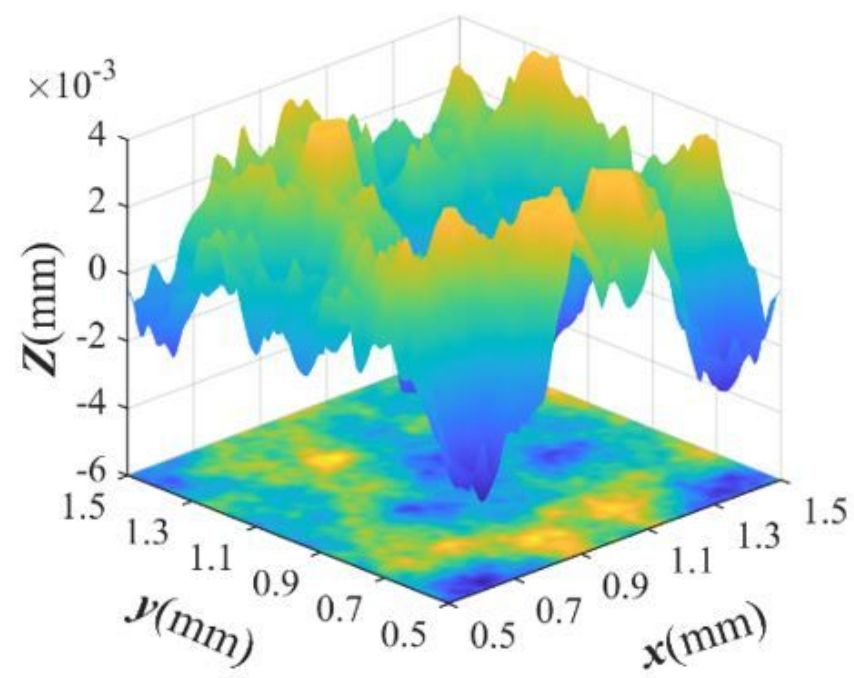

(c) $\mathrm{n}_{\max }=8$

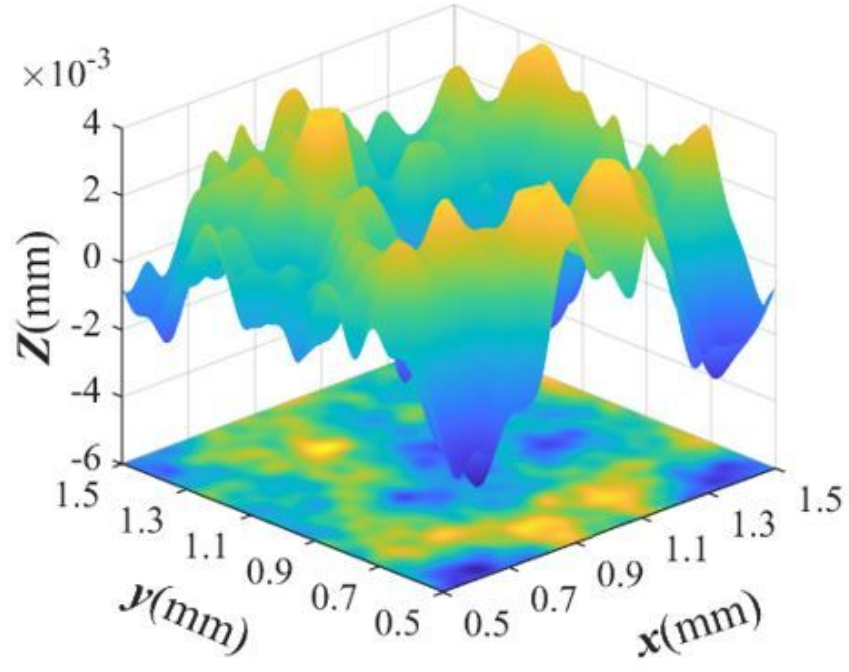

(b) $n_{\text {max }}=6$

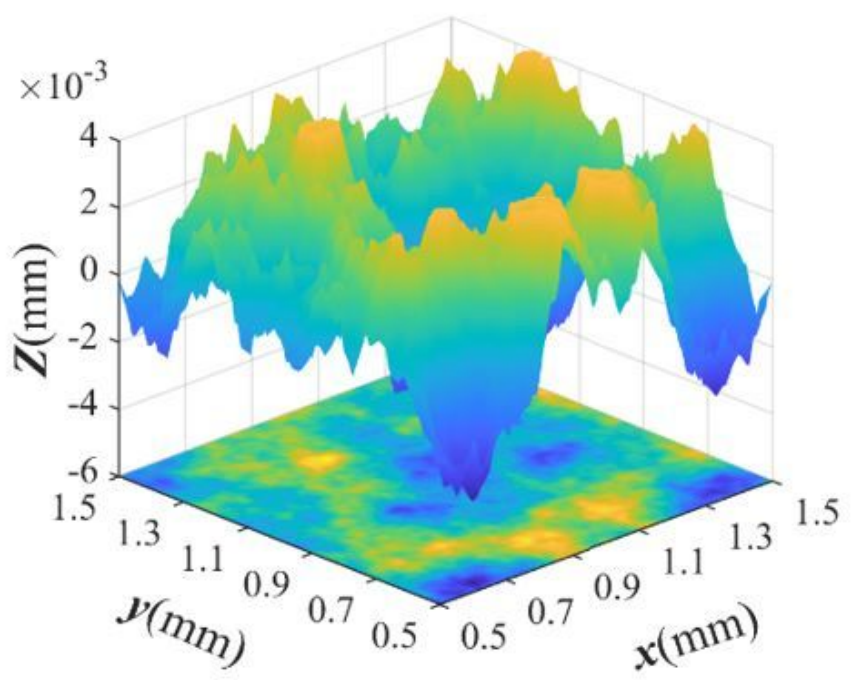

(d) $n_{\max }=20$

Figure 1

Surface topographies for $D=2.3, G=1.5 \times 10-10 \mathrm{~mm}, \mathrm{~L}=1 \mathrm{~mm}$, and $M=6$ 


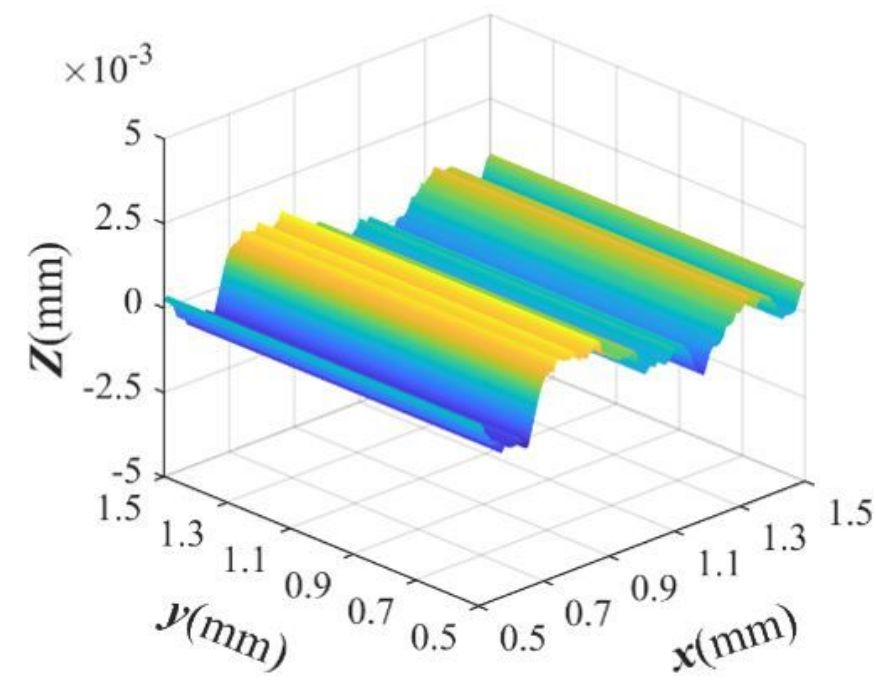

(a) $\mathrm{M}=1$

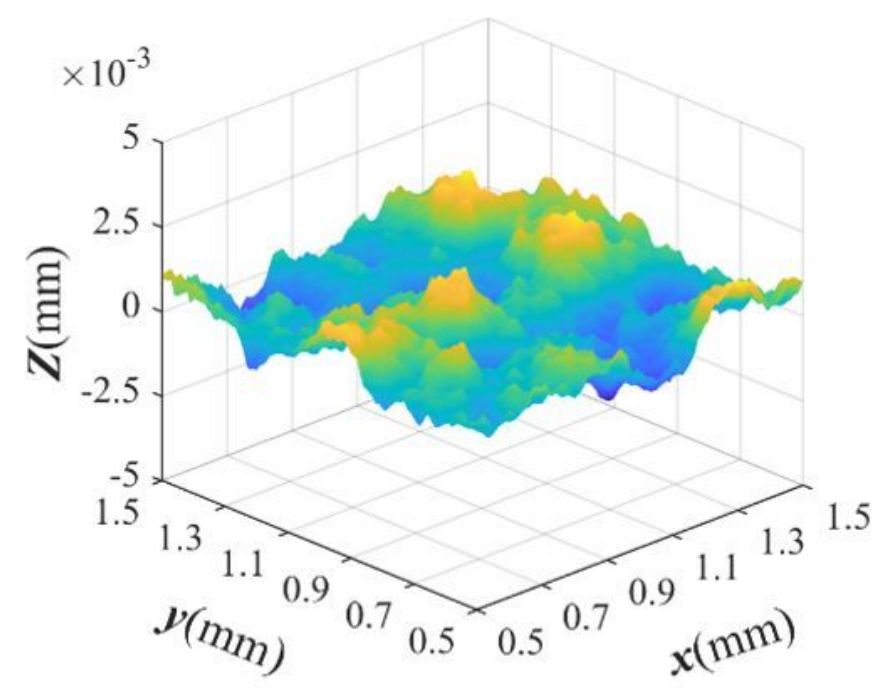

(c) $M=4$

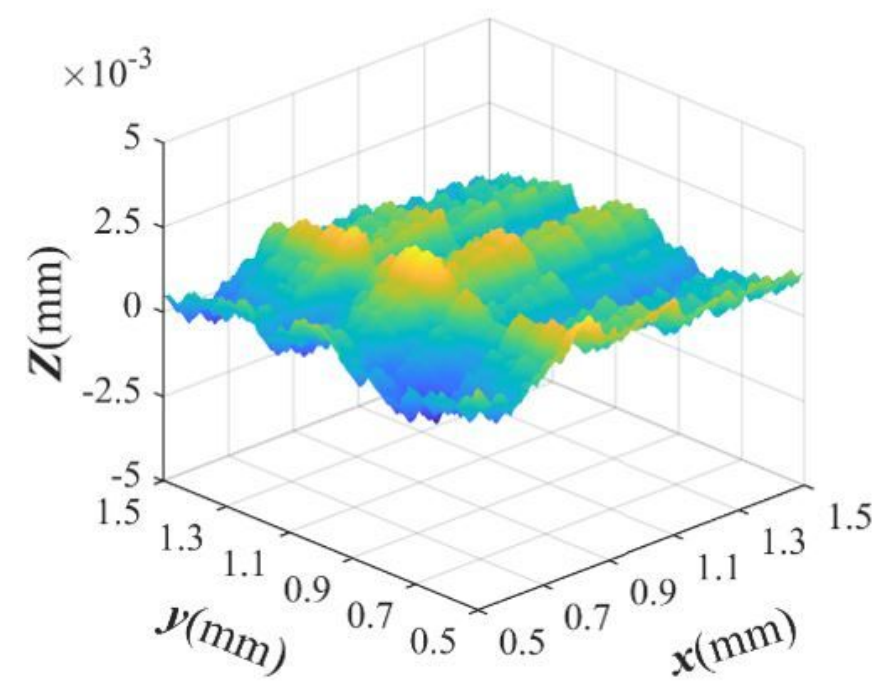

(b) $M=2$

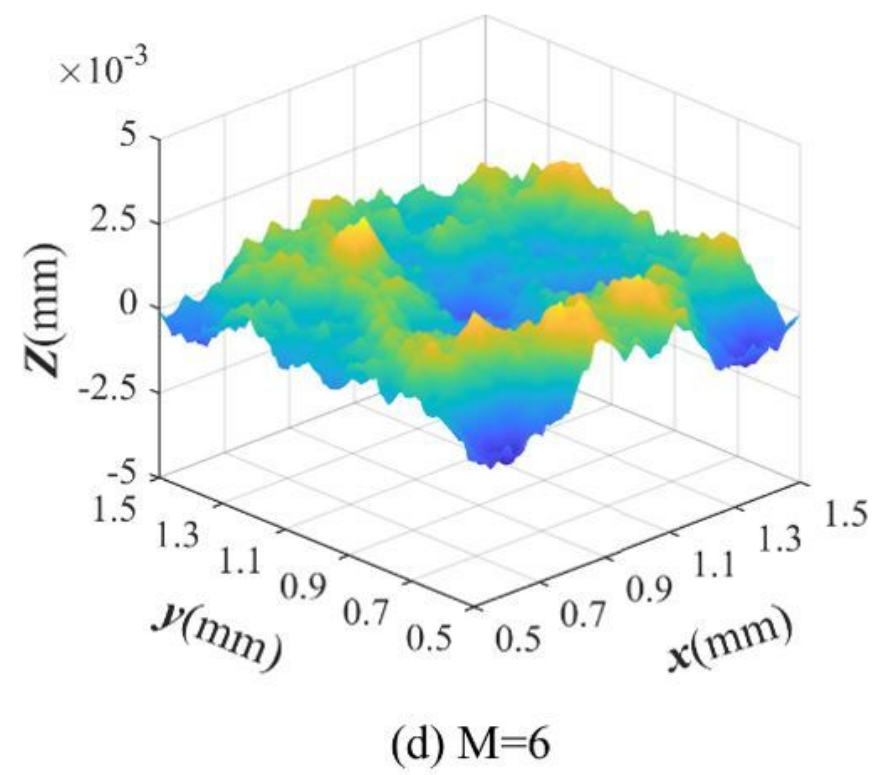

\section{Figure 2}

Surface topographies for $D=2.3, G=1.5 \times 10-10 \mathrm{~mm}, \mathrm{~L}=1 \mathrm{~mm}$, and $\mathrm{nmax}=34$ 


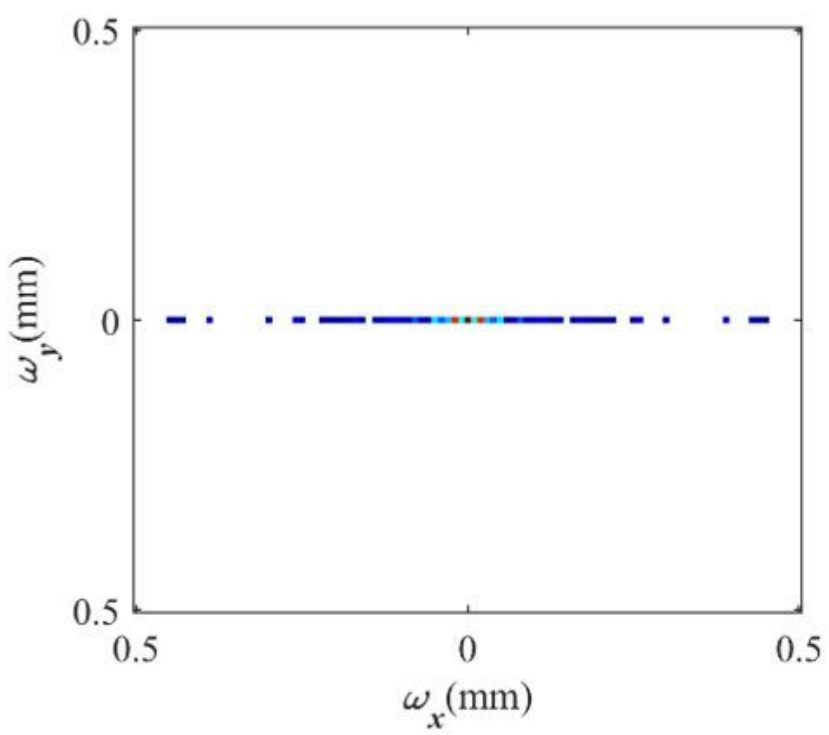

(a) $\mathrm{M}=1$

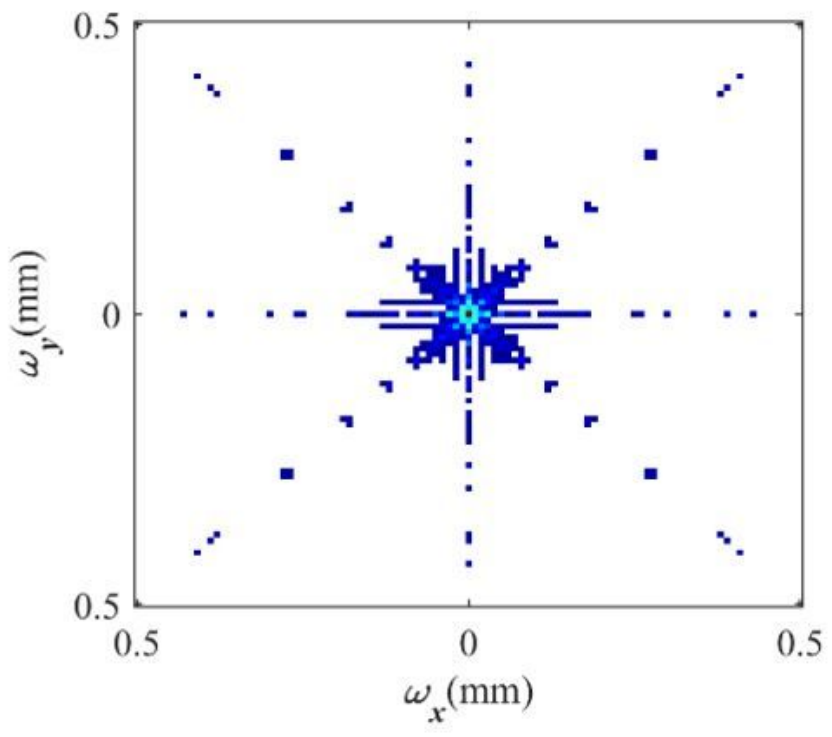

(c) $M=4$

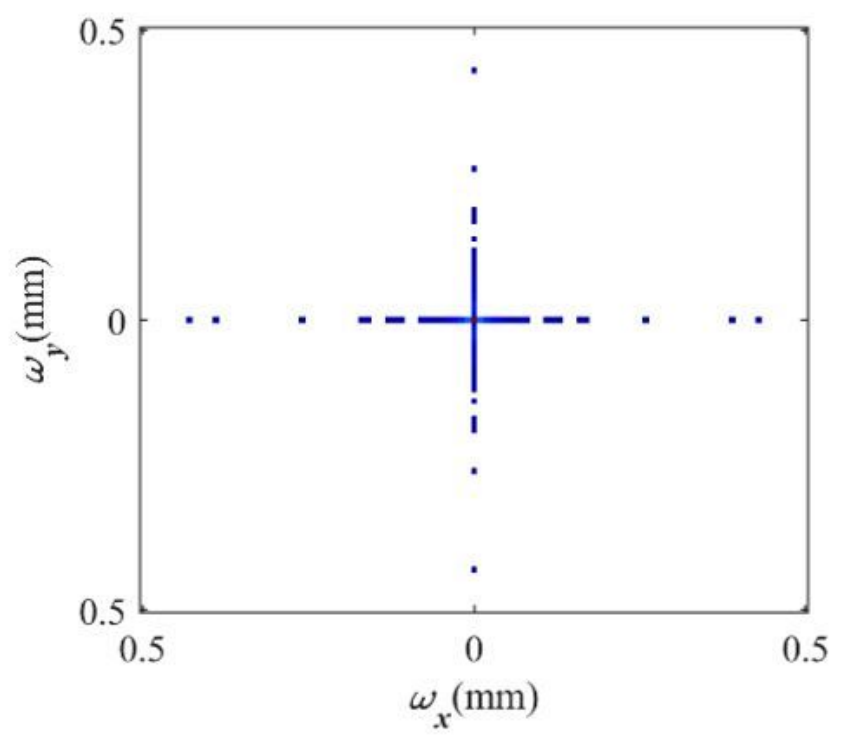

(b) $\mathrm{M}=2$

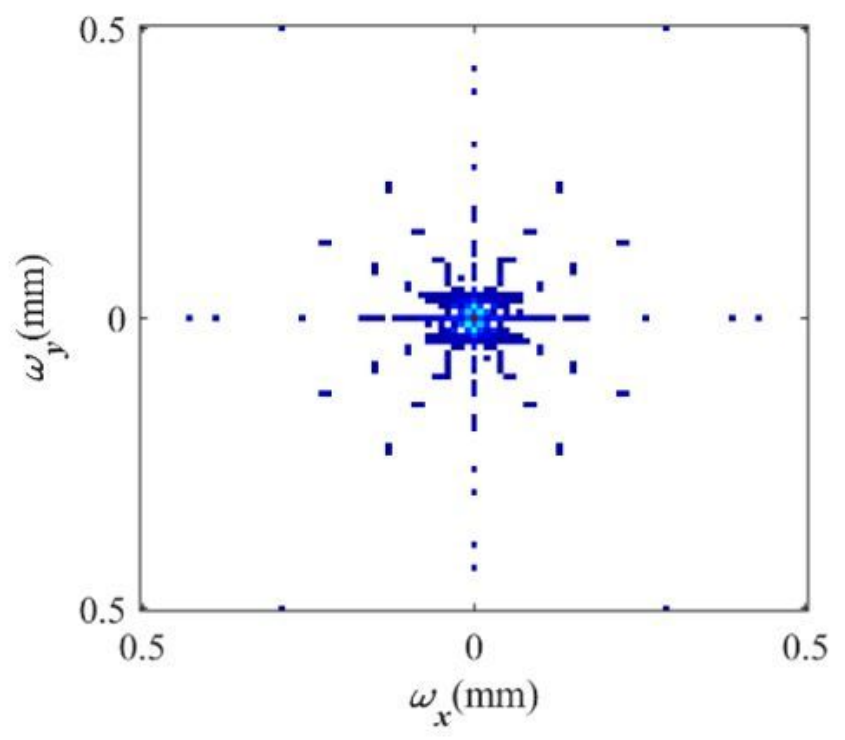

(d) $M=6$

Figure 3

Contour graphs of fractal surface topography power spectra 


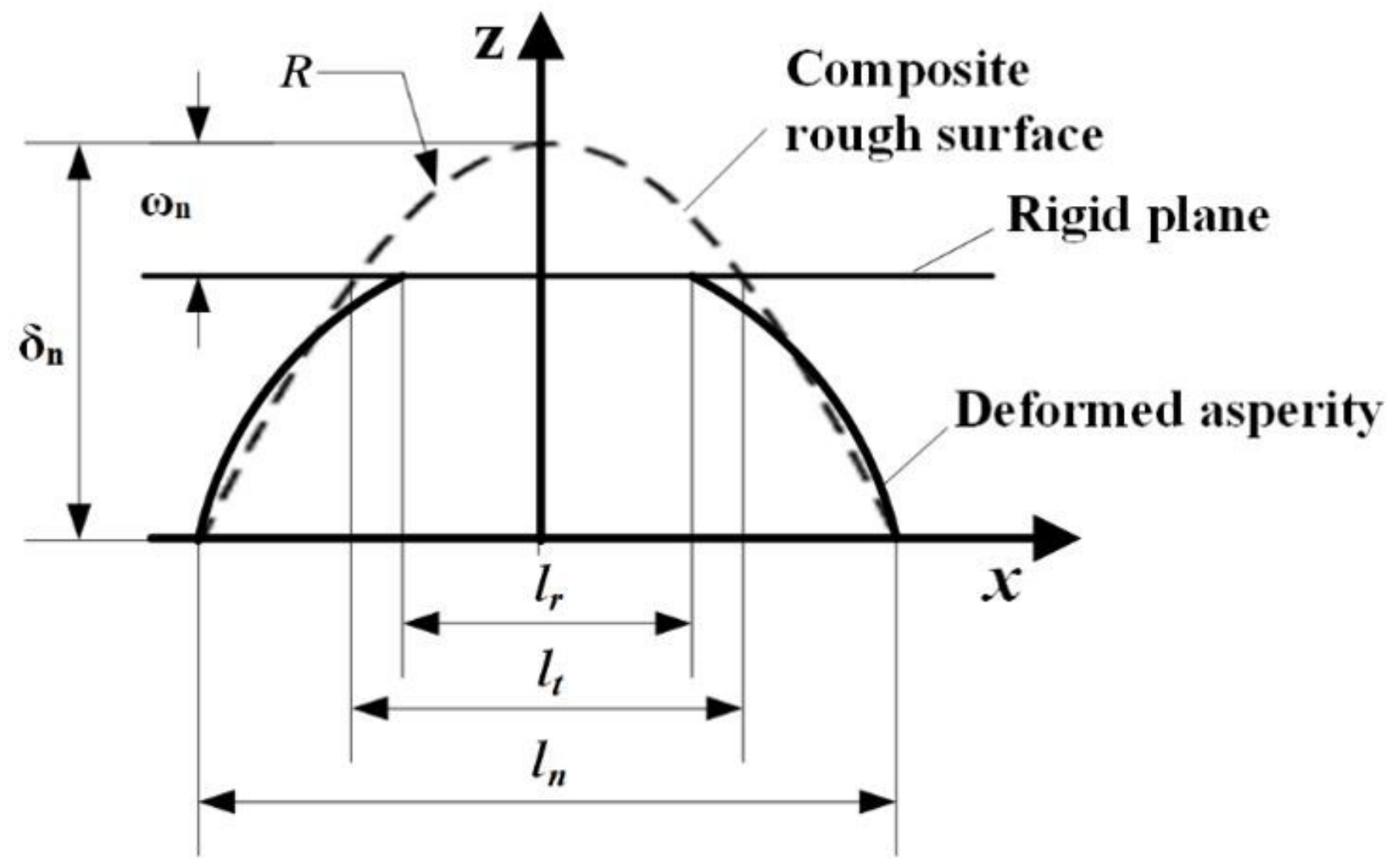

Figure 4

Schematic diagram of geometry of contact asperity with level nn. 


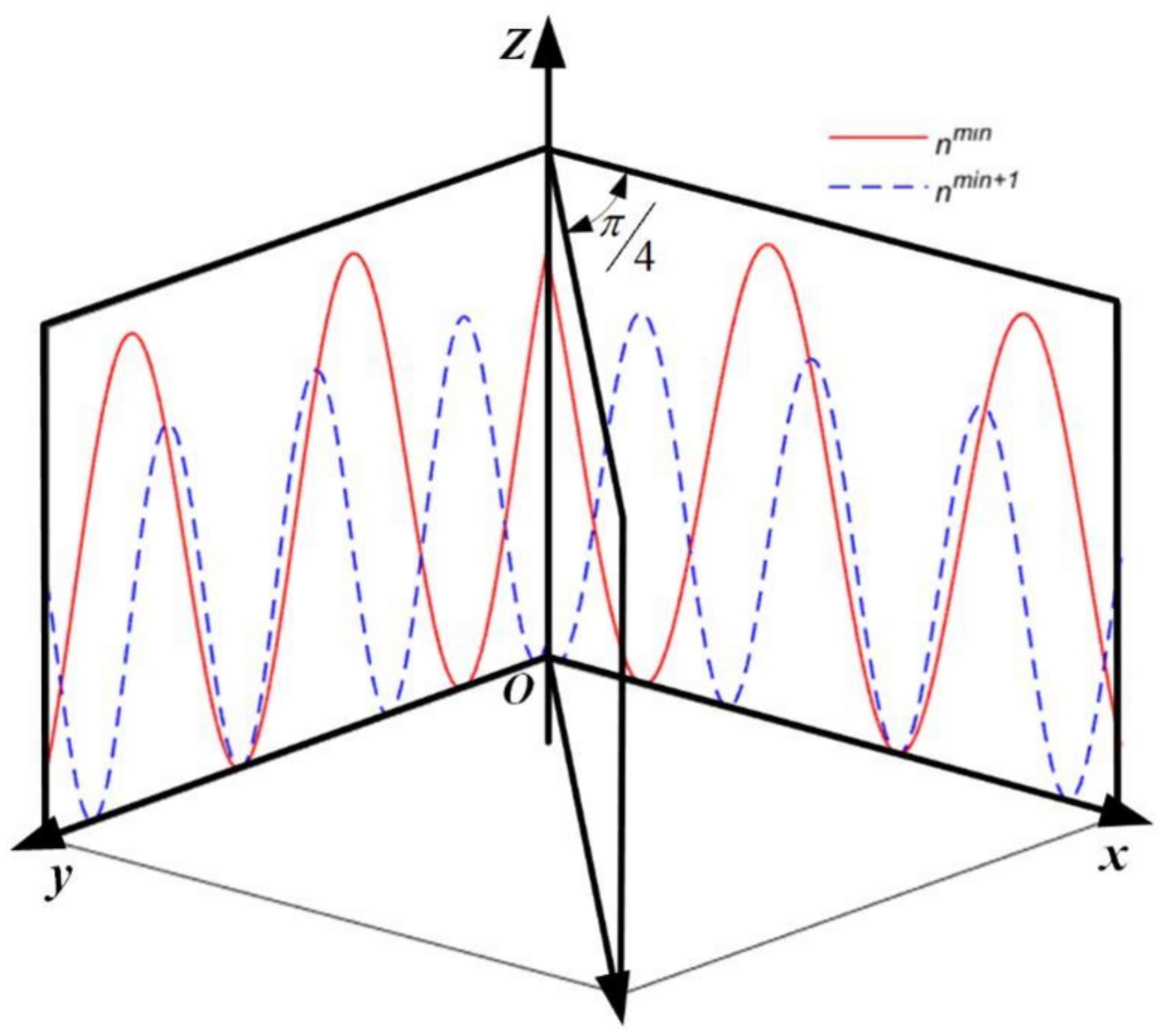

Figure 5

Schematic diagram of simplified three-dimensional surface model 


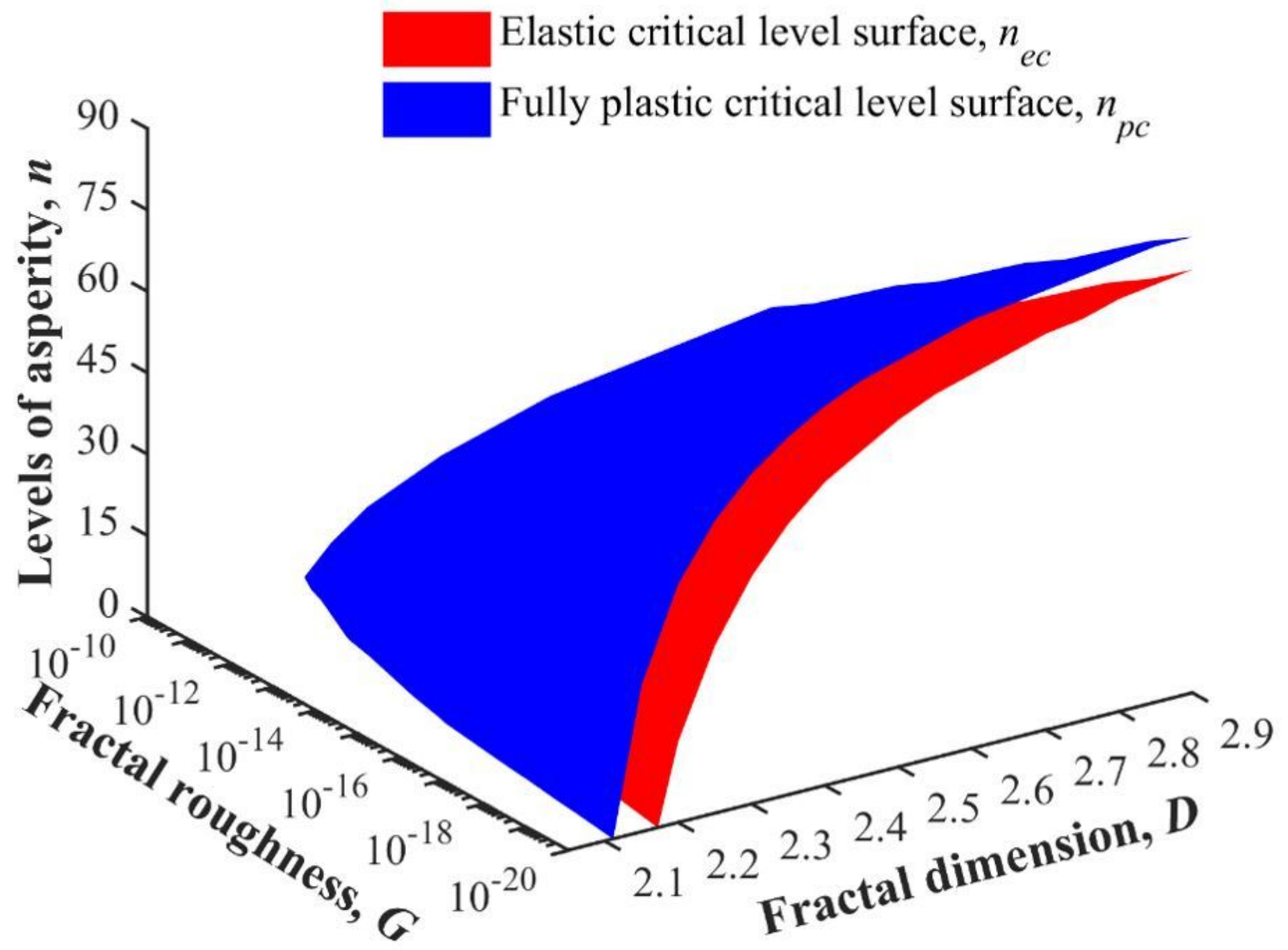

Figure 6

Relationship among fractal roughness, fractal dimension and critical asperity levels. 


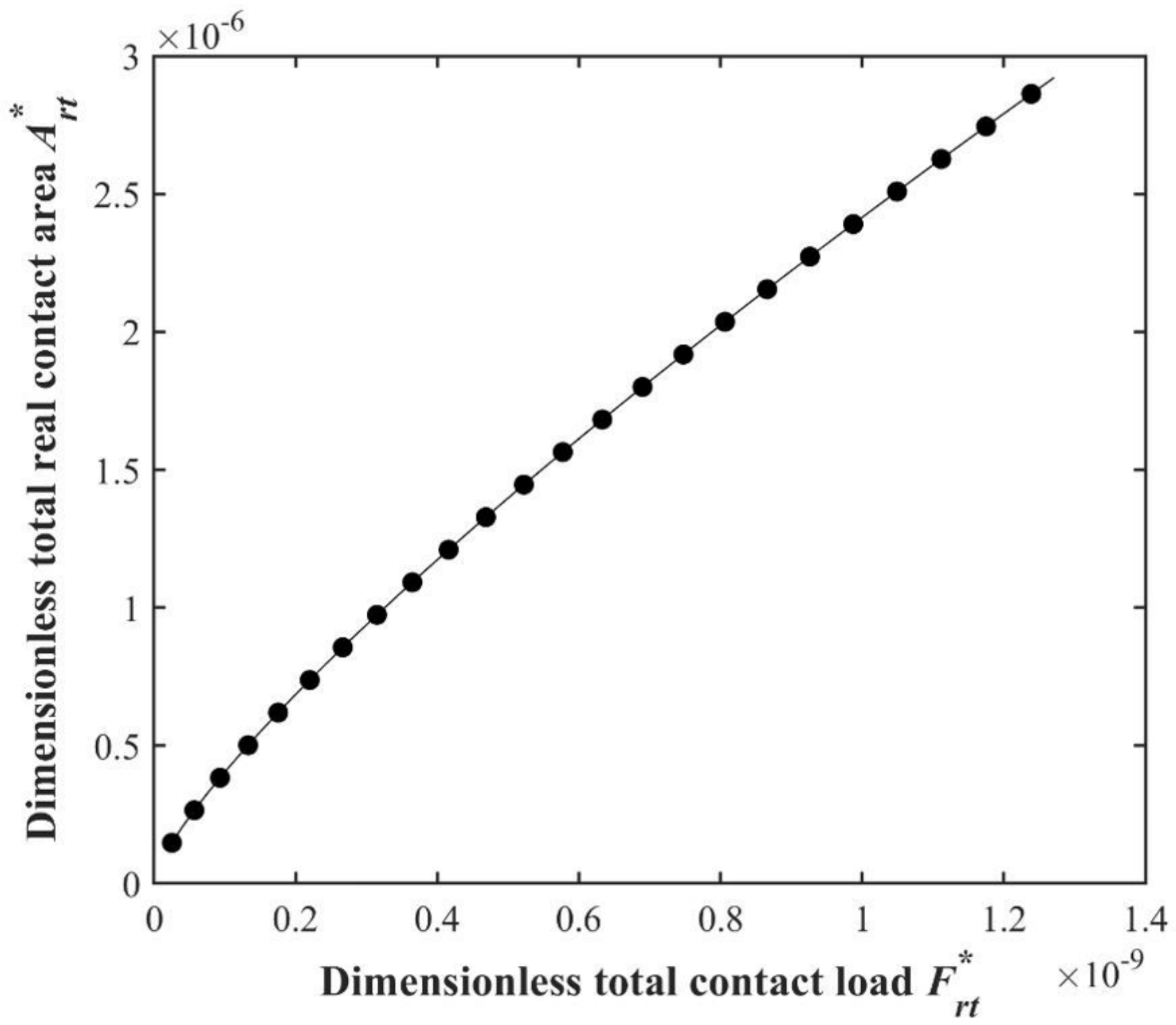

Figure 7

Total nondimensional actual contact area vs. nondimensional contact load for $n \min =17$ and $\mathrm{nmax}=56$ 


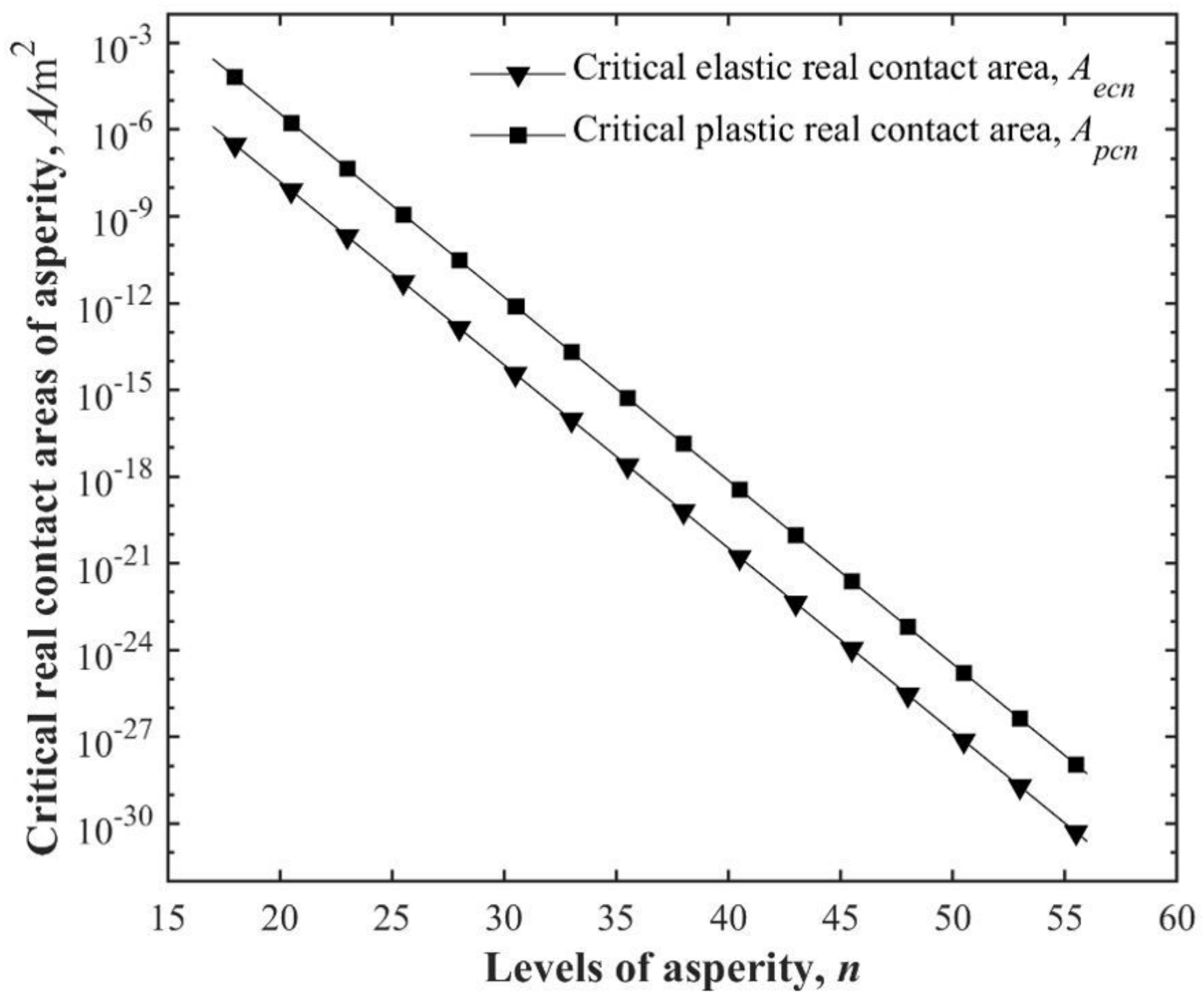

Figure 8

Critical actual contact areas vs. levels of asperity 


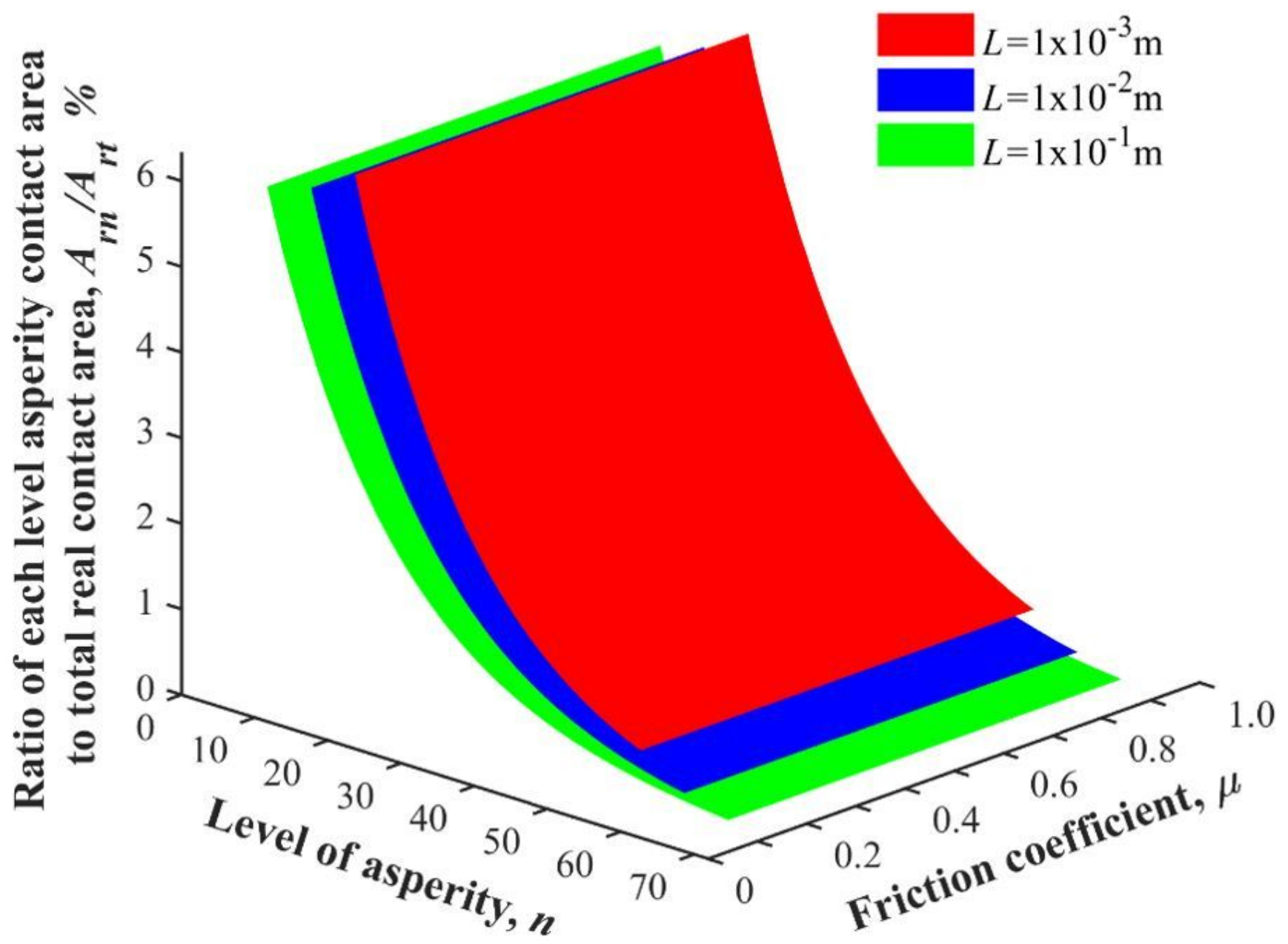

Figure 9

Ratio of each level asperity microcontact area to total actual contact area. 


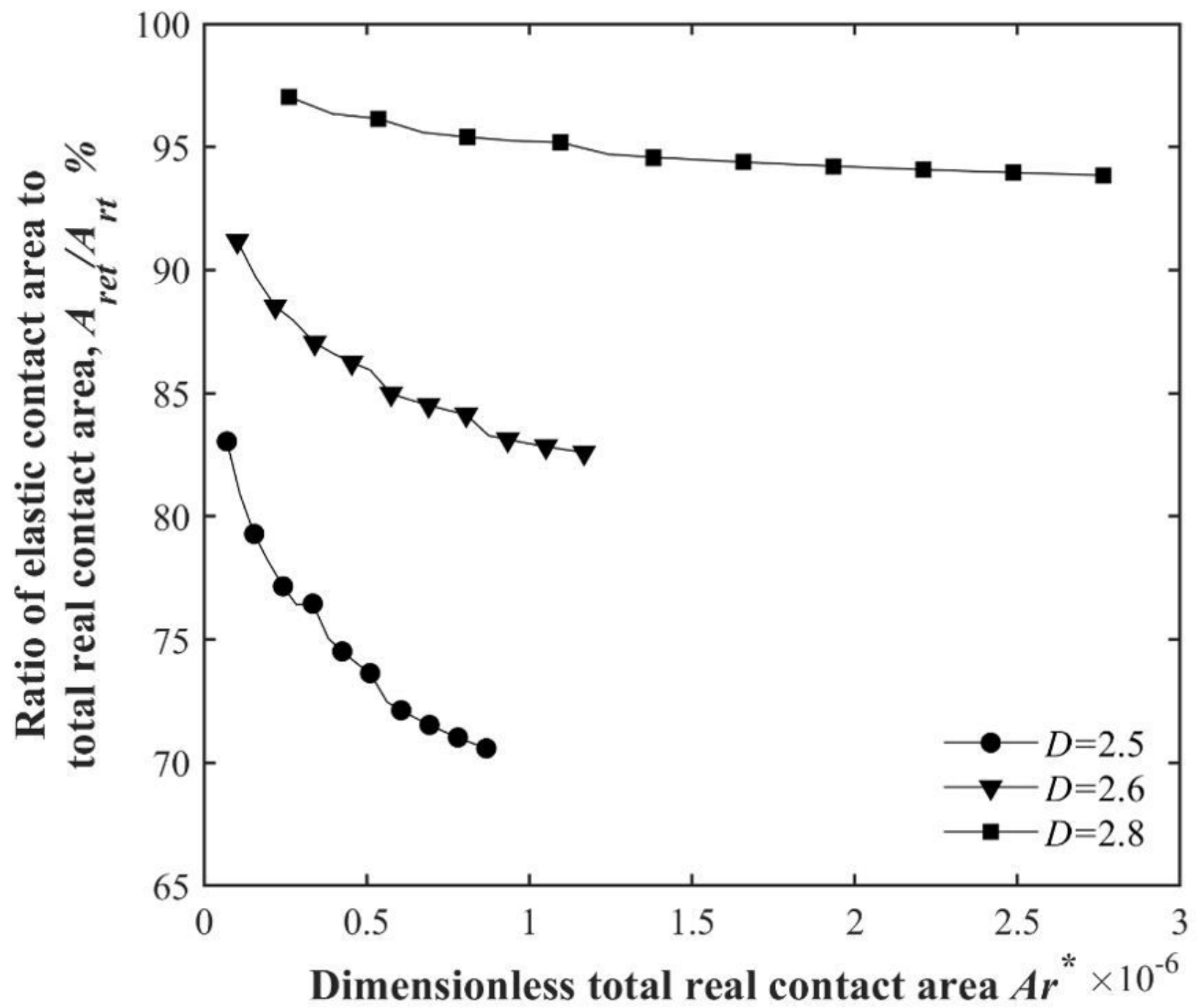

Figure 10

Ratio of elastic contact area to total actual contact area. 


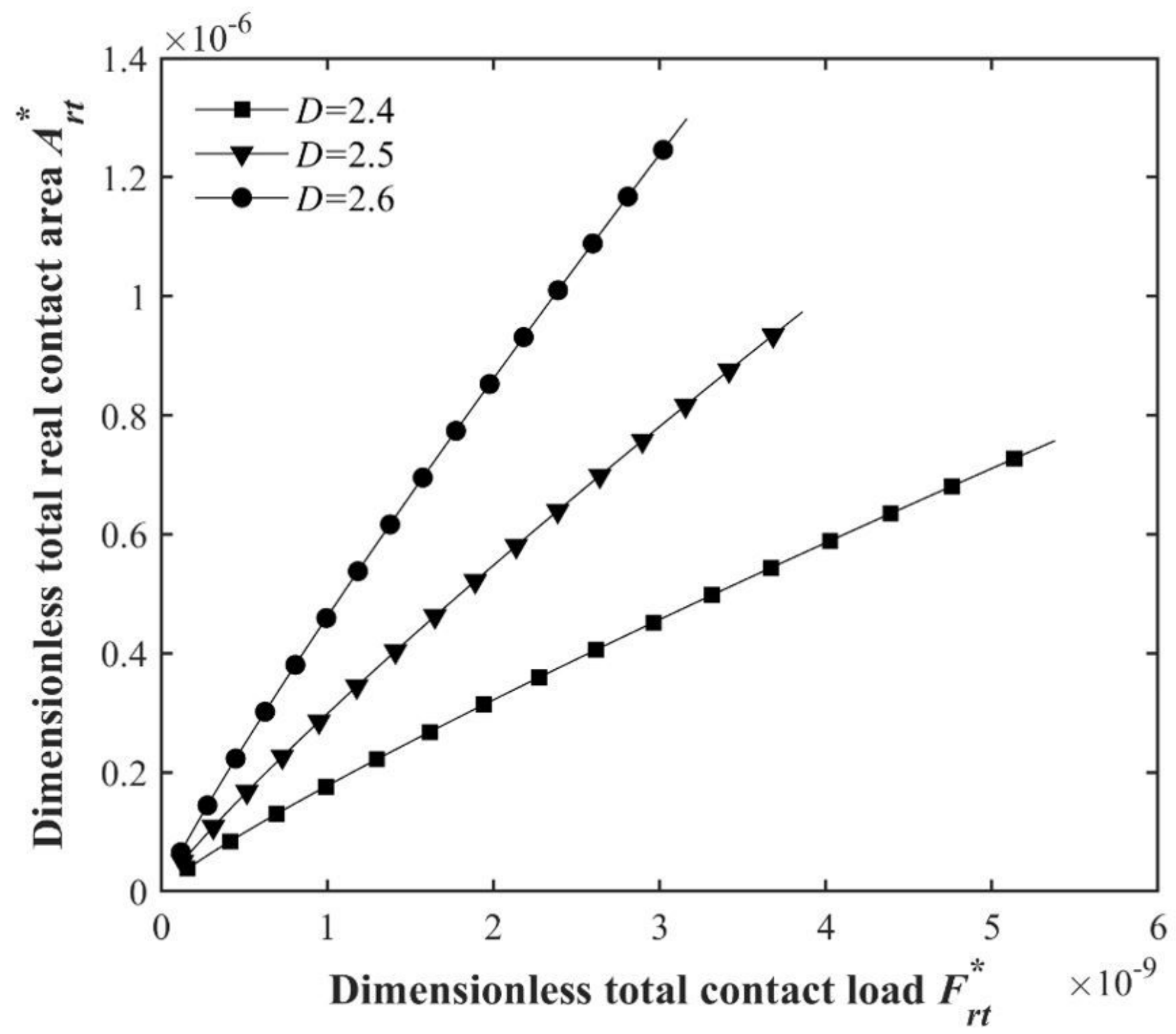

Figure 11

Relationships among nondimensional total contact area, fractal dimension and nondimensional total contact load for $\mathrm{nmin}=17$ and $\operatorname{nmax}=56$. 


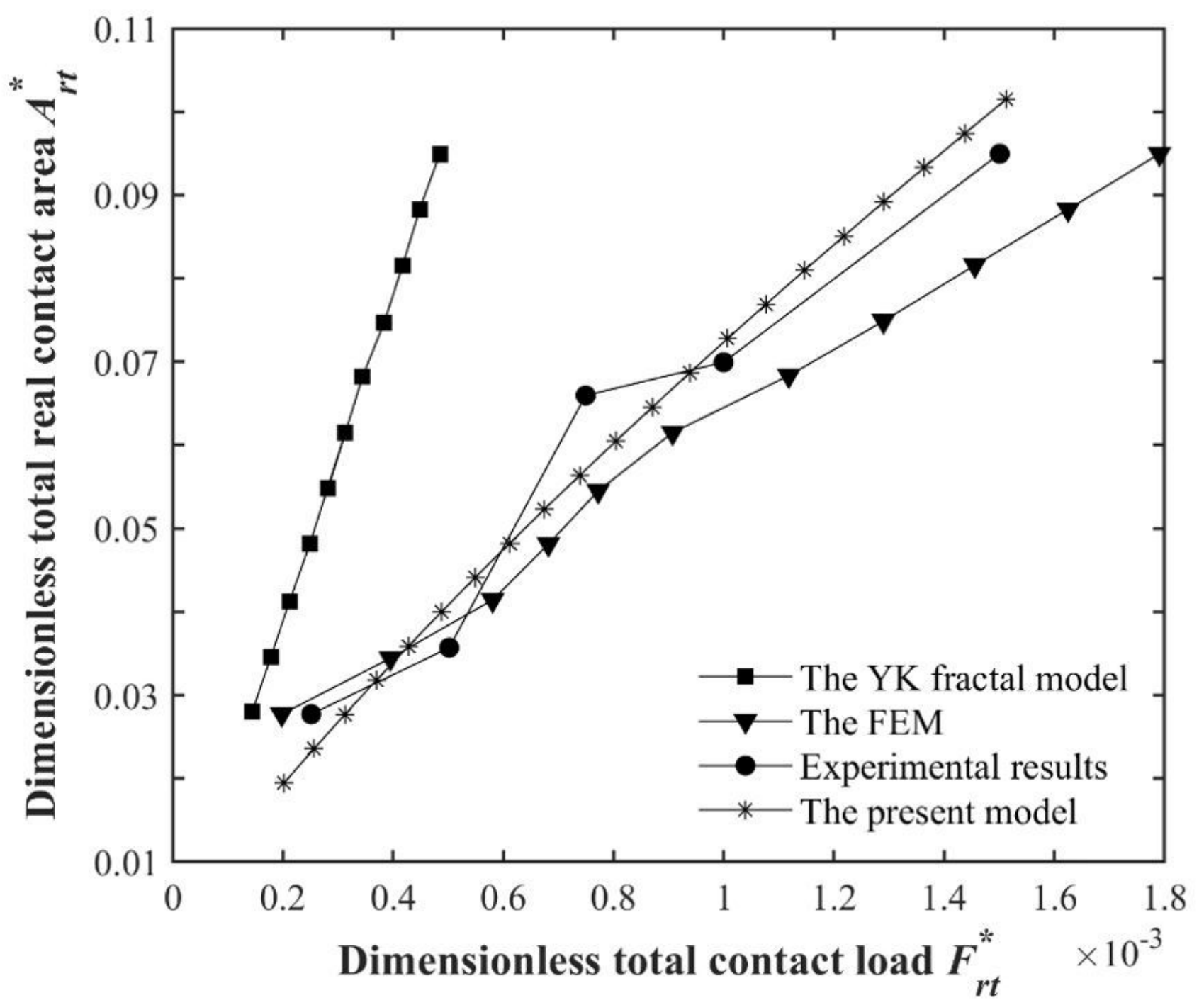

Figure 12

Comparison of proposed fractal model, YK fractal model, and FEM model with experimental results.

\section{Supplementary Files}

This is a list of supplementary files associated with this preprint. Click to download.

- EDITORIALCERTIFICATE.pdf 